\title{
Contexte socio-institutionnel et économique et développement de l'entrepreneuriat scientifique au
}

\section{Cameroun}

\section{Socio-institutional and economic context and development of scientific} entrepreneurship in Cameroon

\author{
Marcel Dama Dié ${ }^{1}$, Martin Fabrice Edou Asséko ${ }^{1}$ \\ ${ }^{1}$ ESSEC, Université de Douala, Cameroun
}

\begin{abstract}
RÉSUMÉ. L'objectif de cet article est d'identifier les facteurs qui inhibent les efforts gouvernementaux qui concourent au développement de l'entrepreneuriat scientifique au Cameroun. La mobilisation des sources documentaires et les données statistiques obtenues à l'Institut National de la Statistique (INS) et dans certains ministères ont permis de conclure que l'objectif de développement de l'entrepreneuriat scientifique au Cameroun est inhibé à la fois par un difficile environnement social, institutionnel, structurel et économique. Pour réduire ces freins, il est premièrement suggéré au gouvernement d'actualiser le Plan Directeur de la recherche existant, dans le but d'identifier et de circonscrire les domaines prioritaires de la Recherche pour le développement du pays ; deuxièmement d'élaborer des stratégies visant à vulgariser auprès des populations la culture de la science, de la technologie et de l'innovation ; troisièmement de mettre en place une structure d'intermédiation entre la recherche et le monde des entreprises pour favoriser la valorisation industrielle et commerciale des résultats scientifiques et technologiques; et enfin accroitre la capacité des institutions de formation et de recherche en infrastructures pédagogiques et d'accueil.

ABSTRACT. The objective of this paper is to identify factors that inhibit government efforts that contribute to the development of scientific entrepreneurship in Cameroon. The mobilization of documentary sources and the statistical data obtained from the National Institute for Statistics (INS) and certain ministries have made it possible to conclude that the objective of developing scientific entrepreneurship in Cameroon is inhibited by a combination of a difficult social, institutional, structural and economic environment. To reduce these obstacles, it is firstly suggested that the government update the existing Research Master Plan with the aim of identifying and circumscribing the priority areas of research for the development of the country, secondly to develop strategies aimed at popularizing the culture of science, technology and innovation among the population; thirdly, to set up an intermediation structure between research and the business world to promote the industrial and commercial promotion of scientific and technological results; and finally to increase the capacity of training and research institutions in educational and reception infrastructures.
\end{abstract}

MOTS-CLÉS. Contexte socio-institutionnel, contexte économique, entrepreneuriat scientifique, institutions de recherche, innovation.

KEYWORDS. Socio-institutional context, economic context, scientific entrepreneurship, research institutions, innovation.

JEL CLASSIFICATION : L22, L29, L32, D02, E24.

De nos jours, les pays développés tout comme les pays en développement encouragent l'innovation en tant que moteur du développement socioéconomique [IND 2019]. En effet, avec le développement des $\mathrm{TIC}^{1}$, il est désormais admis que l'innovation est présente dans tous les domaines de l'économie. Ces technologies occasionnent la diffusion rapide des techniques de production et de marchandisation des biens de consommation courante et durable [FLI 2016]. De plus, les technologies numériques associées à ces TIC facilitent les processus de marchandisation et d'industrialisation des biens culturels et artistiques en incorporant celles-ci comme élément de design des autres types de biens, améliorant de ce fait leur image de marque [BOU 2010]. Ainsi, l'innovation n'est plus seulement présente dans les entreprises de haute technologie et les secteurs technologiques. Ce qui semble d'ailleurs l'argument essentiel qui anime les pays à mettre résolument l'accent sur la création et le maintien d'écosystèmes et de réseaux d'innovation fiables et dynamiques en leur sein.

\footnotetext{
${ }^{1}$ Technologies de l'Information et de la Communication
} 
En d'autres termes, il s'agit pour les pays, au nord comme au sud, de mettre en place un cadre propice à l'entrepreneuriat scientifique ; c'est-à-dire un environnement qui contribue à stimuler les comportements, motivations et désirs de création de technologies nouvelles ou de découvertes scientifiques. Cela renvoie aussi à la capacité des gouvernants à inciter et à motiver les universités à produire des biens et services commercialisables dans le but de financer l'innovation en leur sein [GAS 2003]. Généralement, cet environnement est non seulement composé des écoles et universités spécialisées ayant mis sur pied des programmes de formation adaptés qui favorisent l'esprit de créativité, de recherche et d'adoption de nouvelles découvertes [COU 2015] ; [MBA 2012] ; [MOT 1998] ; [BEL 2018]. Mais aussi, d'un cadre socioéconomique qui fait référence à la culture, à l'éducation, à la pauvreté, à la capacité à s'approprier la technologie à travers l'achat des brevets d'invention et des licences, etc, bref, au niveau de développement économique [GUE 1994] ; [RAH 2011]. En un mot, la capacité à développer l'entrepreneuriat scientifique et technique dans un pays est étroitement liée à son contexte socioéconomique [OCD 2012]. Cette réalité de désir croissant des pays pour financer l'entrepreneuriat scientifique est visible dans les faits stylisés relatant les efforts qu'ils mènent.

Selon le rapport de [IND 2019], «les dépenses mondiales dans le domaine de la recherchedéveloppement ont augmenté plus rapidement que l'économie mondiale et ont plus que doublé entre 1996 et 2016 ». Toujours, d'après la même source, «en 2017, les dépenses publiques mondiales en recherche-développement ont augmenté d'environ 5\%, tandis que les dépenses des entreprises en recherche-développement ont augmenté de 6,7\%, la plus forte hausse depuis $2011 »$. Cependant d'après le World Development Indicators [WDI 2020], les niveaux de financement en recherchedéveloppement sont plus élevés dans les pays développés que ceux en développement. En effet, les pays développés qui sont des pays à revus élevés, à l'instar de la France, le Danemark, l'Allemagne, la Suisse, le Japon, la Suède, la Corée du Sud consacrent à plus de 2,145\% de leur PIB aux activités de recherche-développement, taux équivalent à la moyenne mondiale.

Par contre, selon cette même source, les parts de budget consacrées à la recherche-développement dans l'ensemble des pays d'Afrique Subsaharienne, d'Afrique du Nord et d'Amérique Latine et Caraïbes, considérés comme les moins avancés, s'élèvent respectivement à $0,47 \%, 060 \%$ et $0,71 \%$. Il s'agit des pays dont les budgets sont largement faibles comparativement aux pays développés suscités. Cette incapacité à financer la recherche-développement serait due à la fragilité de ces économies. Par exemple, si dans un pays développé comme la France, l'Etat et les entreprises parviennent à financer les activités de recherche et développement à plus de $50,2^{2}$ milliards d'euros depuis l'année 2015 [MES 2019] $]^{3}$, ce n'est pas le cas pour les pays en développement et, particulièrement pour les pays d'Afrique subsaharienne dont les entreprises sont en majorité de petites tailles. Celles-ci exercent pour la plupart dans l'informel. L'Etat semble donc incapable de collecter suffisamment les recettes pour soutenir les activités de recherche et développement.

Face à cette réalité, le gouvernement camerounais bien qu'ayant développé des partenariats en matière de coopération universitaire pour l'élaboration d'un écosystème d'entrepreneuriat scientifique, se trouve confronté à d'autres réalités de nature socioculturelle. En effet, les bailleurs de fonds constitués pour l'essentiel des organismes multilatéraux (UNESCO, Banque Mondiale, FMI), s'intéressent plus à financer l'enseignement primaire et maternel que l'enseignement supérieur qui est le socle de l'entrepreneuriat scientifique. D'après ces institutions, les taux de déperdition scolaire sont élevés au niveau de l'enseignement primaire ; constituant de ce fait un frein majeur pour le développement économique des pays en développement en général, et particulièrement du Cameroun. De plus, même en présence des systèmes de coopération établis avec des organismes tels que l'Institut Régional de Coopération Développement (IRCOD) et la Fondation Internationale pour la Science (FIS), les universités camerounaises n'arrivent pas à développer

\footnotetext{
2 Dont 32,6 milliards d'euros réalisés par les entreprises et 17,6 milliards par les administrations.

${ }^{3}$ Ministère de l'enseignement supérieur, de la recherche et de l'innovation, France.
} 
l'entrepreneuriat scientifique [DAM 2019]. Cette observation amène à identifier dans le contexte social, institutionnel et économique de ce pays ; les facteurs qui inhibent le développement de l'université entrepreneuriale au Cameroun. Il s'agit de rechercher d'une part les raisons d'ordre social, culturel et institutionnel et, d'autre part les freins d'ordre économique qui expliquent l'incapacité des universités camerounaises à stimuler en leur sein la recherche et le développement, la production des biens et services et à inculquer l'esprit de créativité aux étudiants.

La méthodologie suivie à cet effet a consisté à collecter des informations issues des sources documentaires ; des données statistiques sur la situation de l'enseignement supérieur au Cameroun ; disponibles à l'Institution National de la Statistique (INS), au Ministère de l'Enseignement supérieur (MINESUP) et d'autres ministères et institutions chargés de la formation professionnelle. Pour parvenir à cet objectif, quatre points sont développés. Le premier présente la revue de littérature sur la relation entre le contexte socio institutionnel et économique et, le développement de l'entrepreneuriat universitaire. Le second expose les effets du contexte socio institutionnel des universités sur leur incapacité à développer l'entrepreneuriat scientifique. Le troisième analyse les effets du contexte économique sur le développement de l'entrepreneuriat scientifique. Et le quatrième présente quelques cas de succès de l'entrepreneuriat scientifique malgré un environnement de recherche pénible.

\section{Revue de la littérature}

L'idée que le cadre économique et le contexte socio-institutionnel, notamment les organes et les règles qui structurent une activité, influent sur le résultat des agents est désormais admise dans la littérature économique [KOL 2006]. Dans ce sillage, [GAS 2003] montre que les prédispositions entrepreneuriales sont le fruit de l'évolution des agents économiques dans des milieux donnés. Cette réalité socioéconomique et institutionnelle, présente dans la littérature en entrepreneuriat a été traitée dans le cadre de l'entrepreneuriat scientifique par les auteurs [FLO 99], [KIT 05]. Pour mieux comprendre cela, il convient de visiter, d'une part, les travaux portant sur la théorisation du concept d'entrepreneuriat scientifique et; d'autre part de présenter quelques exemples factuels issus de certains pays.

\subsection{La théorisation dans le domaine de l'entrepreneuriat scientifique}

Le concept d'entrepreneuriat scientifique est né de l'évolution et de la transformation sans cesse de l'institution universitaire [BOU 19]. Selon cette source, cette transformation découle des considérations d'ordre scientifique (production de connaissances), économique (modalités de financement), social (stratification sociale) et politique (rapports de forces politiques) dont l'université n'a cessé de faire face au fil du temps depuis sa création au $13^{\mathrm{e}}$ siècle. Ce concept récent, comme le souligne [UZU 2018] tend à éclairer sur la nouvelle mission assignée à l'université depuis la fin du $20^{\mathrm{e}}$ siècle : elle doit produire des savoirs qui favorisent l'innovation et qui répondent à des besoins solvables. En d'autres termes, au-delà de sa mission de formation, elle doit rendre «nécessaire le développement d'une relation étroite entre la production industrielle et la production de connaissances scientifiques, relation dans laquelle l'entrepreneur fait figure de médiateur » [BOU 19]. Cette façon de voir l'université comme un lieu en perpétuels mouvements, dont le rôle est de satisfaire les besoins de la société selon l'époque considérée n'est pas acceptée de tous.

Certains théoriciens comme [SHI 05] et [LAM 06], soutiennent que la création d'entreprises dans le domaine scientifique est synonyme d'une remise en cause de l'autonomie universitaire. En effet, pour ces derniers, l'université doit demeurer «un système social normé, autonome, stratifié, disciplinairement segmenté, et différencié des autres sphères professionnelles» [SHI 05]. Selon cette thèse, l'université doit être enfermée hermétiquement dans ses préoccupations scientifiques. Elle ne doit avoir aucun lien avec la société. Par contre, d'autres théoriciens [COM 03], [ETZ 08] 
prennent appui sur les thèmes qui se rapportent à «l'esprit d'entreprise » ou au vœux «d'adaptation des chercheurs » au monde économique moderne pour revendiquer l'étroite relation entre l'université et la production industrielle. D'après ceux-ci, depuis le $13^{\mathrm{e}}$ siècle l'université est résolument tournée vers les besoins de la société. Son évolution et sa transformation ont conduit à l'émergence d'une « technoscience » qui est à l'origine de la rupture des frontières qui séparaient la recherche et la sphère économique.

Ainsi, la création d'entreprises dans le domaine scientifique doit, au regard de l'évolution actuelle du monde, constituer une condition sine qua none pour le développement économique des pays. Mais pour que le développement de l'entrepreneuriat scientifique soit effectif au sein des universités, des conditions socio-institutionnelles et économiques doivent être remplies. Ces conditions peuvent être résumées en cinq points essentiels ${ }^{4}$ :

1- La création d'un cadre de recherche et développement approprié. C'est-à-dire un espace partagé qui est dédié au développement de projets de recherche collaboratifs dans les différentes filières scientifiques proposées par l'université

2- La mise en place au sein de l'université d'un organe constitué d'experts en protection de la propriété intellectuelle. Cet organe devra guider les étudiants et chercheurs inventeurs dans la conception et l'exécution d'une stratégie de protection de leur invention ou résultat de recherche scientifique. Cette protection pouvant prendre la forme d'un brevet, de design industriel, de droits d'auteur ou d'une marque de commerce.

3- La construction d'un cadre de maturation scientifique et technologique des projets constitués d'experts spécialisés dans le développement d'innovations de pointe. Ces experts doivent être, chargés d'accompagner les étudiants et chercheurs inventeurs dans la maturation de leur projet à toutes les étapes. Ils doivent aussi être chargés d'accompagner ceux-ci dans le processus de transfert des résultats de recherche, technologique ou de connaissance à une entreprise ou dans un milieu social. Ils doivent enfin être chargés d'identifier des cibles commerciales et faciliter les mises en relations au sein de l'université avec les responsables chargés de conclure les contrats d'exploitation.

4- La création d'un pôle d'accompagnement, de sensibilisation et de repérage dont le but est d'identifier les étudiants ayant la fibre intrapreneuriale et ou entrepreneuriale et qui sont porteurs de projets d'innovation. Les experts de ce pôle doivent ensuite stimuler, chez les chercheurs-inventeurs, des aptitudes visant à accélérer l'émergence, le développement et le déploiement d'innovations technologiques et sociales responsables, à partir de leurs résultats de recherche.

5- Enfin la mise en place d'un pôle prospection dont le rôle est d'accompagner les chercheursinventeurs et les étudiants chercheurs à l'évaluation des besoins financiers de leurs projets et la recherche du financement requis.

Au-delà de ces facteurs institutionnels, l'environnement social constitue aussi un déterminant pour la réussite et le développement de l'entrepreneuriat scientifique. En effet, à la suite de [FLO 09], [KIT 05] en s'interrogeant sur la possibilité à "encourager les universités à exploiter leur potentiel dans un environnement social complexe et multidimensionnel de création de connaissances qui s’inscrit dans une économie du savoir mondialisée », montre que cela n'est possible que si «les communautés qui gravitent autour de l'université » sont en "mesure d'absorber et d'exploiter les connaissances scientifiques, les innovations et les nouvelles technologies qui sont le fruit de ses activités». Ce qui sous-tend que, comme dans le cas de l'entrepreneuriat classique confère [DRU 1985] et [FOR 2018]; l'émergence d'une économie d'entrepreneuriat scientifique dans une communauté est à la fois influencée par des événements culturel, psychologique et économique ou technologique. Ce qui semble être la raison pour laquelle

\footnotetext{
${ }^{4}$ https://www.ulaval.ca/entrepreneuriat-responsable/entrepreneuriat-scientifique-et-innovant
} 
certaines universités réussissent facilement à implémenter en leur sein des projets d'entrepreneuriat scientifique alors que d'autres n'y parviennent pas [KRI 19].

\subsection{Quelques exemples factuels dans des pays}

[MAT 2015] montre que, suite à l'émergence de l'entrepreneuriat scientifique, consécutive à la mise en place des espaces collaboratifs d'innovation par les grandes universités américaines, de nombreux pays élaborent des politiques de soutien à ce type d'activité. Dans la même visée, [BEL 2018] montre que depuis que le Maroc a adopté une politique visant à intégrer au sein des universités les nouveaux canaux de l'enseignement de l'entrepreneuriat, à savoir la sensibilisation, la formation et l'accompagnement cela a suscité l'esprit d'entreprendre au sein des étudiants ; puis déclenché une prise de conscience pour la création et la production des biens au sein des universités, améliorant de ce fait leurs ressources financières.

D'autres auteurs [AUE 2003] ; [LOC 2005] qui se sont intéressés à analyser ces actions politiques des Etats, retiennent plusieurs facteurs qui stimulent l'entrepreneuriat scientifique. Ainsi, [AUE 2003] montre que la réussite en matière d'entrepreneuriat scientifique est due à la capacité des pays à se doter d'une invention brevetable ou d'un logiciel, donc à faciliter en son sein des transferts de technologies. Cela engendre une expertise technique, un conseil ou un savoir-faire au sein des étudiants et enseignants. Ce qui est à l'origine de la créativité et de l'innovation technologie à l'université. D'autres études [MAR 2005]; [O'SH 2005]; [WRI 2005] traitant du contexte américain, montrent que les centres d'incubation créés au sein des universités apparaissent d'autant plus efficaces dans la mise sur pied de l'entrepreneuriat scientifique qu'ils détiennent des compétences techniques, industrielles, juridiques, de ressources financières, des connaissances par rapport au monde des affaires et d'expérience dans l'élaboration de bons réseaux d'affaires. Dans ces conditions, ces centres aident les universités non seulement à créer, innover ou inventer des produits nouveaux mais aussi à se rapprocher du marché.

Dans une étude portant sur la Wallonie en Belgique, [BEG 2019] pointe comme principaux freins à la création d'entreprises au sein de l'Université, les facteurs tels qu'un environnement difficile en termes d'éducation, d'accès aux financements et aux marchés, de lourdeur administrative et de crainte des sanctions en cas de faillite, ainsi qu'un état d'esprit ambiant qui ne reconnaît et ne récompense pas suffisamment l'esprit des porteurs de projets. Il montre aussi que le déficit de culture entrepreneuriale constitue aussi un frein au déclenchement de l'entrepreneuriat scientifique. Pour pallier cela, il propose une prise de conscience de l'ensemble des États membres de l'Union européenne. Corroborant les idées présentes dans le plan d'action ${ }^{5}$ de cette institution, il montre que la réussite de l'entrepreneuriat scientifique dans un pays «repose sur le développement de l'éducation et de la formation en entrepreneuriat, la création d'un environnement économique favorable, la mise en avant de modèles à suivre et l'inclusion de groupes spécifiques ».

Dans la même logique, [OCD 2012] montre que la Tunisie, jadis caractérisé par un tissu économique composé d'entreprises traditionnelles, à faible croissance et aux faibles opportunités de génération de revenus, a pu légèrement améliorer ses conditions économiques ces 10 dernières années en introduisant dans son système éducatif des programmes et des réformes pour stimuler l'entreprenariat au niveau national et local et; en facilitant l'accès au financement par la mise en place de nouveaux fonds de capital d'amorçage et les garanties de prêts. Aussi a-t-elle déployé un système de soutien des start-up par la création de nouvelles pépinières d'entreprises, la formation et le coaching de porteurs de projet, le but étant d'améliorer leurs qualifications et leurs compétences dans leur secteur d'activité. Aujourd'hui, selon cette source la quasi-totalité des universités et universités de sciences appliquées de ce pays ont développé en leur sein leurs propres activités pour l'éducation entrepreneuriale et le soutien aux start-up ; c'est-à-dire des incubateurs.

\footnotetext{
${ }^{5}$ Commission Européenne (2013) « Plan d'action « Entrepreneuriat 2020. Raviver l'esprit d'entreprise en Europe », Bruxelles.
} 
Au regard de ce qui précède, les conditions sociales, institutionnelles et économiques susceptibles de déclencher un développement de l'entrepreneuriat scientifique peuvent être identifiées non seulement dans le cadre de fonctionnement des universités animé par des comportements humains et sociaux ; mais aussi dans l'environnement économique du Cameroun.

\section{Un environnement social et institutionnel difficile pour le développement de l'entrepreneuriat scientifique}

Plusieurs facteurs sociaux et institutionnels expliquent les difficultés à développer l'entrepreneuriat scientifique au Cameroun. [DAM 2019], dans une étude portant sur les facteurs explicatifs des traits caractéristiques de la relation entre université et les entreprises, détaille ces différents facteurs en les classant en aspects réglementaires et environnementaux des universités. Cependant, il ne traite pas de la difficulté de coordination de la recherche scientifique au Cameroun face aux multiples institutions qui interviennent dans ce domaine qu'il convient d'étayer ici en premier lieu.

\subsection{La recherche scientifique face à un mécanisme national de coordination inefficace}

L'arsenal juridique qui encadre le fonctionnement du système de recherche scientifique au Cameroun est constitué de plusieurs textes élaboré chacun pour une institution donnée. En effet, il existe au Cameroun plusieurs institutions ${ }^{6}$ qui œuvrent dans le domaine de la recherche scientifique. Ce qui est à l'origine des difficultés de coordination de cette activité, même si cette objectif est destinée prioritairement au Conseil de l'Enseignement Supérieur et de la Recherche Scientifique et Technique (CESRST). Concrètement, le CESRST est chargé de l'harmonisation des activités entre les institutions universitaires, d'une part, et les organismes chargés de la recherche scientifique et technique d'autre part; et la participation des opérateurs économiques au développement de l'enseignement supérieur et de la recherche scientifique et technique ${ }^{7}$.

Mais malgré l'existence de cette structure, la coordination du système national de recherche reste inefficace [CIR 2014]. Une des matérialisations de ce manque d'efficacité est l'absence d'un plan directeur national de la recherche, avec pour conséquence une délimitation floue de l'objet et du périmètre de la recherche universitaire. Cette dernière ne peut non plus définir, de manière isolée, sa propre stratégie, en raison de la suspension de l'élaboration des stratégies à périmètre ministériel par le gouvernement. De ce fait, l'entrepreneuriat scientifique universitaire manque d'un ancrage national qui aurait pu baliser son cadre de déploiement. A cela s'ajoute un certain attentisme face à l'idée que c'est le Ministère de la Recherche Scientifique et de l'Innovation qui est la pointe du gouvernement en matière d'entrepreneuriat scientifique. Aussi est-il difficile, dans un contexte de fortes restrictions budgétaires, à la représentation nationale d'aménager un espace budgétaire supplémentaire à la recherche universitaire après avoir « satisfait » les exigences du Ministère de la Recherche Scientifique et de l'Innovation.

On peut également rajouter le cloisonnement des différents acteurs et des doublons. Dans un tel contexte, l'utilisation sous optimale des ressources est inévitable. Plusieurs raisons expliquent cette efficacité limitée: les sessions du Conseil de l'Enseignement Supérieur et de la Recherche Scientifique et Technique sont rares, le leadership du Ministère de la Recherche Scientifique et de l'innovation est, à peine accepté et la capacité de coordination du système par le MINRESI est sujet

\footnotetext{
${ }^{6}$ Parmi ces institutions, il y a : - le Conseil de Recherche Scientifique, premier organe de coordination et d'orientation de la recherche, rattaché directement à la Présidence de la République ; - l'Office National de la Recherche Scientifique et Technique (ONAREST) rattaché au Ministère de la Recherche Scientifique ; - le Conseil de l’Enseignement Supérieur et de la Recherche Scientifique et Technique, rattaché au Ministère de l'Enseignement Supérieur.

${ }^{7}$ Article 2 du décret n99-201 du 17 septembre 1999 portant organisation et fonctionnement du Conseil de l’Enseignement Supérieur et de la Recherche Scientifique et Technique.
} 
à caution [MIN 2014]. [MBO 2004] vont plus loin en attribuant cette inefficacité de coordination à la dépendance du système éducatif à ceux des pays colonisateurs (France, Grande Bretagne, Allemagne). D'après ces auteurs, les axes de recherche scientifique, ses objectifs et son financement répondent prioritairement aux besoins extérieurs, c'est-à-dire de ces pays. Toutefois, il faut souligner que contrairement à ces auteurs, les difficultés relatives au développement de l'entrepreneuriat scientifique sont davantage à souligner au niveau managérial.

\subsection{Des freins d'ordre managérial dans un cadre réglementaire dispersé entre plusieurs institutions}

En termes d'aspects réglementaires de l'encadrement de l'entrepreneuriat scientifique au Cameroun, il est démontré que le cadre de fonctionnement des universités est constitué d'intéressantes et ambitieuses dispositions juridiques, mais qui sont confrontées à de nombreux freins d'ordre managérial. En effet, malgré des réformes visant à garantir une formation de qualité, il faut relever que la politique nationale de développement de la recherche scientifique et, plus précisément du développement de l'entrepreneuriat scientifique, continue à subir de nombreux problèmes de gouvernance ${ }^{8}$.

[KEM 2012] montre que ces difficultés sont dues aux pesanteurs d'une bureaucratie lourde et autocentré, à une insuffisance de ressources financières et une faiblesse d'esprit d'entreprise. Selon ce dernier, leur origine découle ; d'une part du caractère flou de la réforme universitaire en matière de répartition des ressources entre le rectorat et les différentes composantes de l'université, puis entre les chefs d'établissements et les départements. D'autre part, d'un manque d'une parfaite collaboration entre les responsables des différentes institutions de recherche scientifique (MINESI, MINESUP, CESRST, ONAREST) ; malgré des dispositions juridiques de coordination qui existent.

S'agissant de la collaboration au sein des universités, il faut noter que leur fonctionnement est basé sur des circulaires, directives et arrêtés dont le but est d'équilibrer les ressources et les responsabilités entre le rectorat et les établissements comme souligné dans la loi portant orientation de l'enseignement supérieur. Cependant, ces instruments juridiques fournissent des effets mitigés ; car les recteurs, en se fondant sur l'argument selon lequel ils sont nommés par le président de république, évitent de les appliquer en exhibant la supériorité des décrets qui les ont portés à leur poste de responsabilité. Malgré les normes universitaires de 2015 applicables aux établissements de l'enseignement supérieur dans le but d'éradiquer ces comportements des dirigeants des universités, ce phénomène perdure. Pourtant ces normes précisent clairement les rôles que doivent jouer ceux-ci en matière de gouvernance administrative, financière, numérique, sociale, heuristique et de partenariat. Leur objectif étant de rendre les établissements universitaires financièrement autonomes et de leur garantir un cadre de travail adéquat. A travers elles, le gouvernement a également pour souci d'encourager ces établissements à créer en leur sein des formations continues, des laboratoires de recherche, de souscrire à des polices d'assurances pour les enseignants, de créer des partenariats avec les entreprises ou bien de créer des entreprises qui pourront vendre les résultats des recherches universitaires, etc... Ces comportements des dirigeants de l'enseignement supérieur semblent constituer un frein qui décourage le développement et la concrétisation des projets rentables au sein de l'université et par conséquent la mise sur pieds d'une université entrepreneuriale.

Concernant le manque de collaboration entre les responsables des différentes institutions dédiées aux activités de recherche scientifique, il faut noter que cette négligence est la cause des dysfonctionnements observés dans l'accueil des stagiaires universitaires dans les instituts de recherche. Ils sont dus aux difficultés dont souffrent les relations entre les Instituts de recherche sous tutelle du MINRESI et les Universités, sous tutelle du MINESUP. Par exemple, le mode de

\footnotetext{
${ }^{8}$ Notion holistique définie par la banque mondiale (1992) dans un rapport comme étant la manière dont le pouvoir est exercé pour gérer les ressources nationales économiques et sociales consacrées au développement.
} 
financement est différent entre ces deux catégories de structures. Les Instituts de recherche sont attributaires des fonds publics sur la recherche tandis que les Universités dont le premier rôle est la formation des étudiants tirent leurs ressources du nombre d'étudiants inscrits. Bien que dans les deux cas, ces ressources financières soient insuffisantes pour le fonctionnement de ces différentes structures, leurs dirigeants ne se déploient pas à mettre en œuvre les dispositions statutaires de collaboration édicté dans le cadre de l'organisation et le fonctionnement du Conseil de l'Enseignement Supérieur et de la Recherche Scientifique et Technique (CESRST). Pour justifier leur refus, ils demandent que les ressources financières qu'ils disposent soient complétés par des financements supplémentaires. Pourtant, dans un pays qui voudrait accélérer son processus de développement, les limites budgétaires ne devraient pas constituer un obstacle pour l'accueil des apprenant dans les structures de formation. Or, face aux ressources financières limitées de l'Etat, ces dirigeants devraient plutôt mutualiser leurs capacités financières. Ce qui constituerait une solution efficace à l'amélioration du cadre de fonctionnement de la recherche scientifique. Au-delà de ces problèmes managériaux, la qualité de l'environnement dans les institutions dédiées à la recherche scientifique semble aussi jonchée des difficultés au développement de l'entrepreneuriat scientifique au Cameroun.

\subsection{Un cadre de fonctionnement des institutions de recherche jonché d'obstacles structurel et socioculturel}

Plusieurs obstacles structurels et socioculturels sont à l'origine du mauvais fonctionnement des institutions de recherche au Cameroun, compromettant de ce fait l'objectif d'entrepreneuriat scientifique que s'est assigné le gouvernement camerounais ${ }^{9}$. Il convient de présenter, d'une part la manières dont ces obstacles se manifestent dans le cadre des universités et, d'autre part dans le cadre des institutions de recherche qui sont sous la tutelle du MINERESI.

\subsubsection{Un environnement universitaire caractérisé par des obstacles structurel et socioculturel}

Les écueils d'ordre structurel et socioculturel présents dans l'environnement interne des universités camerounaises peuvent être résumé en terme de croissance exponentielle de la population estudiantine, de mauvais ratio d'encadrement des étudiants, du manque de filières professionnelles relevant du domaine de l'ingénierie, des industries de transformation et de production, et de manque d'infrastructures d'accueil [DAM 2019].

\subsubsection{Une croissance exponentielle des effectifs d'étudiants}

Le tableau 1 présente cette évolution exponentielle des effectifs d'étudiants. On part de 100196 étudiants dans l'année académique 2004/2005 à 352755 étudiants dans l'année académique $2019 / 2020$ soit une hausse de $252 \%$ en près de 15 ans.

\begin{tabular}{|l|c|c|c|c|c|c|c|c|}
\hline Universités & $2004 / 2005$ & $2009 / 2010$ & $2015 / 2016$ & $2016 / 2017$ & $2017 / 2018$ & $2018 / 2019$ & $2019 / 2020$ & $2019 / 2020$ \\
\hline Publiques & 91334 & 178491 & 214214 & 234569 & 244500 & 269508 & 263779 & 292595 \\
\hline Privées & 8862 & 30551 & 41428 & 50258 & 50558 & 54163 & 58659 & 60160 \\
\hline & & & & & & & & \\
\hline Ensemble & $\mathbf{1 0 0 1 9 6}$ & $\mathbf{2 0 9 0 4 2}$ & $\mathbf{2 5 5 6 4 2}$ & $\mathbf{2 8 4 ~ 8 2 7}$ & $\mathbf{2 9 5 0 5 8}$ & $\mathbf{3 2 3} \mathbf{6 7 2}$ & $\mathbf{3 2 2} \mathbf{4 3 8}$ & $\mathbf{3 5 2} \mathbf{7 5 5}$ \\
\hline
\end{tabular}

Source : Annuaire statistiques du MINESUP, éditions de 2005 à 2020

Tableau 1. Evolution des effectifs d'étudiants entre 2004/2005 et 2019/2020

\footnotetext{
${ }^{9}$ Décret $n^{\circ} 2005 / 342$ du 10 septembre 2005 modifiant et complétant certaines dispositions du décret $n^{\circ}$ 93/027 du 19 janvier 1993 portant dispositions communes aux Universités.
} 
Malgré cette hausse vertigineuse qui anéantit les efforts des pouvoirs publics camerounais pour l'amélioration du cadre de formation au Cameroun, d'autres sources d'information semblent sousestimer ces statistiques. Par exemple, d'après la loi de finances de $2017^{10}$, les effectifs des étudiants au Cameroun sont passés respectivement de 288343 en 2013/2014 à 306508 en 2014/2015 pour atteindre 340746 en 2015/2016. Ce qui contribue à détériorer la qualité de formation. Les enseignements sont rarement suivis de cas pratiques du fait de l'incapacité des enseignants à suivre ces effectifs pléthoriques d'étudiants. Les statistiques relatives aux ratios d'encadrement permettent d'étayer cette réalité.

\subsubsection{Des ratios d'encadrement difficiles à améliorer malgré les efforts des pouvoirs publics}

[DAM 2019] a présenté cette situation alarmante, relative à l'incapacité du gouvernement camerounais à améliorer les ratios d'encadrement (étudiants/enseignants) dans les universités camerounaises. Les données statistiques utilisées par ce dernier sont celles de la période allant de l'année académique 2004/2005 à l'année académique 2012/2013. Dans le cadre de cette étude, en revenant sur cet aspect d'une quête d'un bon encadrement des étudiants dans les universités Camerounaises, il réaffirme que toutes les tentatives d'amélioration des ratios d'encadrement, à travers les différents recrutements d'enseignants initiés par l'Etat du Cameroun, la création de nouvelles universités ${ }^{11}$ et l'encouragement à la création des institutions privées d'enseignement supérieur, ne fournissent pas toujours les résultats escomptés. Le graphique 1 illustre cela.

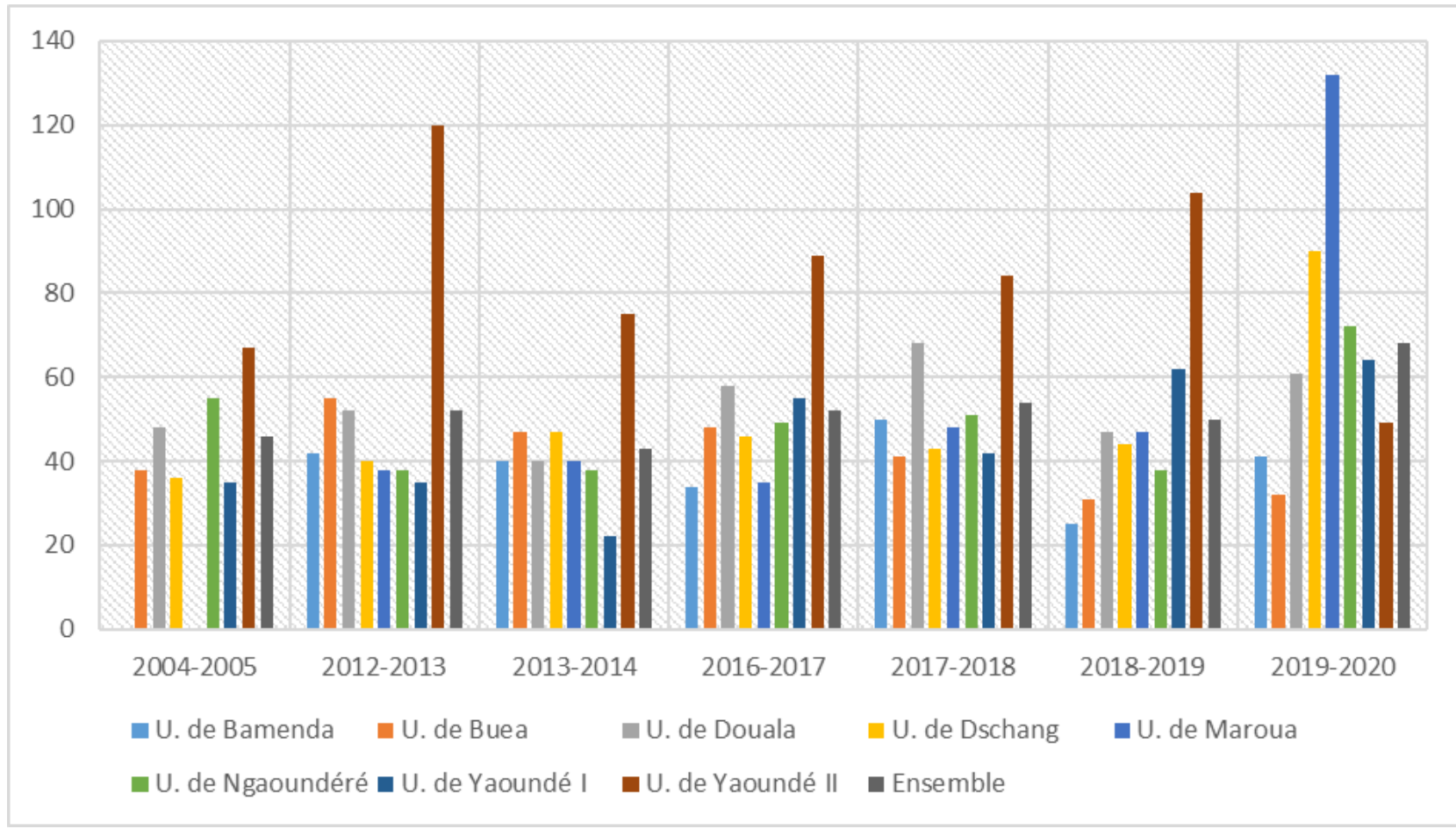

Source : Annuaire statistiques du MINESUP, éditions de 2005 à 2020

Légende : U. de Buea signifie Université de Buea. Ce qui est valable pour les autres cas Graphique 1. Evolution des ratios d'encadrement entre 2004/2005 et 2019/2020

Ce graphique montre par exemple que, pour les universités de Douala, de Yaoundé 2 et de Ngaoundéré les ratio d'encadrement sont respectivement passés de 48, 67, 55 étudiants en 2004/2005 à 52, 120 et 38 étudiants en 2012/2013 pour se situer plus tard à 58, 89 et 49 en 2016/2017, puis 47, 104 et 38 en 2018/2019 et enfin 61, 49 et 72 en 2019/2020. Dans le même

\footnotetext{
${ }^{10}$ Cette loi présente les informations statistiques de tous les domaines régaliens de l'Etat sur lesquels elle se fonde.

${ }^{11}$ Université de Maroua en 2008 et celle de Bamenda en 2010.
} 
temps, le ratio des récentes universités de Maroua et Bamenda est passé de 40 étudiants par enseignant dans chacune des deux universités pour se situer à respectivement à 132 et 41 étudiants par enseignant. Dans le même temps, ces ratios ne sont pas améliorés pour les anciennes universités dont l'Etat du Cameroun avaient pour ambition de décongestionner. La situation s'y est également dégradée.

Ces ratios ne s'améliorent pas parce que la contrainte budgétaire de l'Etat ne permet pas de remplacer les nombreux enseignants qui vont à la retraite. Cela est également dû à l'augmentation des effectifs de nouveaux bacheliers et les redoublements ${ }^{12}$ suite à un cadre de formation mal adapté. Cette stagnation, voire détérioration des effectifs d'enseignants peut s'observer dans le tableau 3.

Ce tableau illustre l'incapacité des pouvoir publics à mettre en place un cadre propice à l'apprentissage en ce sens que les effectifs des enseignants entre 2012/2013 et 2019/2020 sont stables autour de 4000 enseignants. Sauf en 2017/2018 et 2018/2019 où nous avons respectivement 4682 et 5155 enseignants.

\begin{tabular}{|c|c|c|c|c|c|c|c|}
\hline Années & $\mathbf{2 0 1 2 / 2 0 1 3}$ & $\mathbf{2 0 1 3 / 2 0 1 4}$ & $\mathbf{2 0 1 5 / 2 0 1 6}$ & $\mathbf{2 0 1 6 / 2 0 1 7}$ & $\mathbf{2 0 1 7 / 2 0 1 8}$ & $\mathbf{2 0 1 8 / 2 0 1 9}$ & $\mathbf{2 0 1 9 / 2 0 2 0}$ \\
\hline Prof. & 193 & 205 & 236 & 273 & 387 & 375 & 344 \\
\hline MC & 440 & 469 & 639 & 750 & 934 & 1021 & 839 \\
\hline CC & 1310 & 1433 & 1685 & 1823 & 1945 & 2112 & 1488 \\
\hline ASS, & 2039 & 2171 & 1261 & 1361 & 1267 & 1110 & 1135 \\
\hline ATER & 99 & 110 & 256 & 230 & 149 & 537 & 145 \\
\hline Autres & & & & & & 463 & 279 \\
\hline Ensemble & $\mathbf{4 0 8 1}$ & $\mathbf{4 3 8 8}$ & $\mathbf{4 0 7 7}$ & $\mathbf{4 4 3 7}$ & $\mathbf{4 6 8 2}$ & $\mathbf{5 1 5 5}$ & $\mathbf{4 2 3 0}$ \\
\hline
\end{tabular}

Source : Annuaire statistiques du MINESUP, éditions de 2012 à 2020

Légende : Prof. : Professeur, MC : Maitre de conférences, CC : Chargé de Cours, ASS. : Assistant, ATER : Attaché d'Enseignement et de Recherche.

Tableau 2. Effectifs des enseignants par grade dans les universités d'Etat en 2012 et 2019

Et en 2019/2020 le nombre d'enseignants a chuté à 4 230. Cette chute représente l'effectif d'enseignants retraités, décédés ou ayant abandonné leur poste. Cette situation d'encadrement des étudiants est encore plus mauvaise au niveau du troisième cycle dans la mesure où le nombre d'enseignement de rang magistral (Professeurs et Maitres de Conférences) reste faible au fil du temps.

\subsubsection{Très peu d'étudiants en ingénierie, en industries de transformation et de production}

D'après le tableau 3, le nombre d'étudiants qui fréquentent les filières relevant des domaines d'ingénierie, d'industries de transformation et de production est très faible par rapport à ceux inscrits dans les filières relevant des sciences sociales et commerciales et des sciences de l'éducation.

\footnotetext{
${ }^{12}$ Les effectifs de nouveaux bacheliers pour les année 2016/2017, 2017/2018, 2018/19 et 2019/2020 s'élèvent respectivement à 95 565, 92 016, 109484 et 116 950, largement supérieurs aux nombres de diplômés. Par exemple en 2016 il a été enregistré 40792 diplômés (Annuaire statistique du MINESUP, édition 2017).
} 


\begin{tabular}{|c|c|c|c|c|c|c|c|c|c|}
\hline $\begin{array}{c}\text { Domaine } \\
\text { d'études }\end{array}$ & Education & $\begin{array}{c}\text { Lettres } \\
\text { et arts }\end{array}$ & $\begin{array}{c}\text { Sciences } \\
\text { sociales, } \\
\text { commerces }\end{array}$ & sciences & $\begin{array}{c}\text { Ingénierie, } \\
\text { Industries de } \\
\text { transformation } \\
\text { et de } \\
\text { production }\end{array}$ & Agriculture & Santé & services & Total \\
\hline Publique & 23028 & 53609 & 87340 & 51388 & 20472 & 2857 & 5808 &. & $\mathbf{2 4 4 5 0 2}$ \\
\hline & & & & & & & & & \\
\hline Privées & 714 & 1224 & 24832 & 2390 & 6858 & 166 & 4780 & 9592 & $\mathbf{5 0 5 5 6}$ \\
\hline & & & & & & & & & \\
\hline Total & $\mathbf{2 3 ~ 7 4 2}$ & $\mathbf{5 4 ~ 8 3 3}$ & $\mathbf{1 1 2 ~ 1 7 2}$ & $\mathbf{5 3 7 7 8}$ & $\mathbf{2 7 ~ 3 3 0}$ & $\mathbf{3 ~ 0 2 3}$ & $\mathbf{1 0 ~ 5 8 8}$ & $\mathbf{9 5 9 4}$ & $\mathbf{2 9 5 0 6 0}$ \\
\hline
\end{tabular}

Source : Annuaire statistique du MINESUP, édition 2018

Tableau 3. Nombres d'étudiants par filière dans les Universités publiques et privées en 2017/2018

Ce constat sous-tend que les dirigeants camerounais investissent peu dans la création des filières de formation qui relèvent des domaines de production, de la transformation et la production. Ce type de filières sont moins nombreuses au Cameroun. Ce qui semble constituer l'un des blocages pour la concrétisation de l'objectif gouvernemental d'universités entrepreneuriales. En 2017/2018, il est dénombré 27330 étudiants dans les filières d'ingénierie, d'industries de transformation et production; 3023 en agriculture et 53778 en sciences fondamentales et expérimentales contre 112172 en sciences sociales et commerciales, 23742 en sciences de l'éducation et 54833 en lettres et arts. On observe là que l'université au Cameroun forme essentiellement la main d'œuvre destinée au secteur tertiaire qui en réalité, est un secteur qui n'a pour but que de soutenir le secteur productif dans une économie.

\subsubsection{Un manque d'infrastructures d'accueil}

Le tableau 4 montre que la situation infrastructurelle est mauvaise dans les universités camerounaises. Par exemple, en terme d'amphithéâtres les universités de Bamenda, Douala, Yaoundé I et Yaoundé II possédaient récemment en 2018, respectivement 2, 27, 47 et 15 de capacités totales $950 ; 12683,13868$ et 5411 étudiants, contre des effectifs de 16 294, 49 543, 58 617, 49500 étudiants présents dans ces universités dans la même période. La situation parait plus mauvaise lorsqu'on observe les ratios en terme nombre de blocs ateliers/capacités d'accueil, nombre blocs pédagogiques/capacités d'accueil, nombre laboratoire/capacités d'accueil et surtout salles de TD/capacités d'accueil.

\begin{tabular}{|c|c|c|c|c|c|c|c|c|c|c|}
\hline \multirow{2}{*}{ Universités } & \multicolumn{2}{|c|}{ Blocs Ateliers } & \multicolumn{2}{|c|}{$\begin{array}{c}\text { Blocs } \\
\text { Pédagogiques }\end{array}$} & \multicolumn{2}{c|}{ Laboratoire } & \multicolumn{2}{c|}{$\begin{array}{c}\text { Salles de Travaux } \\
\text { Dirigés (TD) }\end{array}$} & \multicolumn{2}{c|}{ Amphithéâtres } \\
& Nombre & Capacité & Nombre & Capacité & Nombre & Capacité & Nombre & Capacité & Nombre & Capacité \\
\hline Bamenda & 5 & 5178 & - & - & 1 & - & - & - & 2 & 950 \\
\hline Buea & 6 & 2850 & 7 & 1825 & 16 & 780 & - & - & 8 & 4984 \\
\hline Douala & 41 & 1986 & 52 & 19740 & 43 & 2139 & 24 & 1440 & 27 & 12683 \\
\hline Dschang & 3 & 115 & 5 & 673 & 71 & 535 & 10 & - & 15 & 6514 \\
\hline Maroua & - & - & 2 & 2400 & 4 & 600 & - & - & 7 & 1444 \\
\hline Ngaoundérén & 2 & 140 & 77 & 5830 & 28 & 582 & 12 & 267 & 3 & 1900 \\
\hline Yaoundé I & 18 & 1022 & 133 & 9331 & 72 & 1095 & 29 & 1635 & 47 & 13868 \\
\hline Yaounde II & 1 & - & 1 & 8 & - & - & 4 & 34000 & 15 & 5411 \\
\hline Total & $\mathbf{7 6}$ & $\mathbf{1 1 2 9 2}$ & $\mathbf{2 7 7}$ & $\mathbf{3 9 8 0 7}$ & $\mathbf{2 3 5}$ & $\mathbf{5 7 3}$ & $\mathbf{7 9}$ & $\mathbf{6 7 4 2}$ & $\mathbf{1 2 4}$ & $\mathbf{4 7 1 5 4}$ \\
\hline
\end{tabular}

Source : Annuaire statistique du MINESUP, édition 2018

Tableau 4. Distribution des types d'infrastructures académiques par université

Pour des effectifs d'étudiants suscités pour les universités de Bamenda, Douala, Yaoundé I, on a respectivement: 1) 5 blocs ateliers à Bamenda de capacité 5178 étudiants, aucun Bloc pédagogique, 1 laboratoire dont la capacité n'a pas été donnée, aucune salle de TD ; 2) 41 blocs 
ateliers à Douala de capacité 1986 étudiants, 52 blocs pédagogiques 19740 étudiants, 43 laboratoires de capacité 2139 étudiants et 24 salles de TD de capacité 1440 étudiants ; 3) 18 blocs ateliers à Yaoundé I de capacité 1022 étudiants, 133 blocs pédagogiques de capacité 9331 étudiants, 72 laboratoires de capacité 1095 étudiants et 29 salles de TD de capacité 1635 étudiants. Comme la jeune université de Bamenda, celle de Maroua n'a pas de salle de TD ni de blocs ateliers. Ces statistiques expliquent les difficultés des universités camerounaises à développer l'entrepreneuriat scientifique et par conséquent, l'incapacité des étudiants sortis de ces universités à entreprendre dans le domaine productif. Les différents obstacles enregistrés ont engendré diverses difficultés d'ordre socioculturel dans les universités camerounaises qu'il convient de présenter ici.

\subsubsection{Les freins d'ordre socioculturel dans l'environnement des universités}

[DAM 19] présente les freins socioculturels qui contribuent à amplifier l'incapacité des dirigeants des universités à développer l'entrepreneuriat scientifique. Il montre en effet que la question des infrastructures d'accueil dans les universités camerounaises a engendré un désarroi au sein de la communauté universitaire. On y assiste à une crise morale et de vocation du corps enseignant, un recours au tribalisme dans l'exercice des services demandés, la tricherie des étudiants lors des examens, des programmes qui obéissent plus à des exigences administratives qu'aux besoins de la société, la qualité des candidats au recrutement qui baisse sans cesse. Cette triste réalité de l'université au Cameroun est présentée par [TCH 2011] qui a effectué une enquête auprès d'un échantillon de 18 établissements d'enseignement supérieur. Ces auteurs affirment que « face à une situation de crise qui a paralysé la société et l'économie nationale au cours des décennies antérieures, les effectifs pléthoriques dans les établissements universitaires sont accompagnés de nombreux problèmes notamment, un faible niveau d'encadrement des étudiants, une détérioration des conditions de travail et des conditions d'évaluation». Pour être plus explicites, ces derniers montrent qu'au-delà de la question des infrastructures d'accueil, la tricherie à l'université au Cameroun est le reflet des dysfonctionnements de la société caractérisée par des facteurs tels que la corruption et le détournement de deniers publics.

\subsection{Les institutions de recherche sous tutelle du MINRESI face à des obstacles structurel et socioculturel}

En observant le cadre de fonctionnement des institutions de recherche au Cameroun, l'on s'aperçoit que leur environnement structurel et socioculturel est constitué de nombreux obstacles qui ne favorisent pas l'essor de l'entrepreneuriat scientifique.

\subsubsection{Les obstacles structurels dont font face les institutions de recherche}

Sur le plan structurel, il faut d'abord dire qu'au-delà des difficultés de coordination des activités de recherche dues aux multiples organes mis en place par le gouvernement, le souci d'élaboration d'un véritable cadre légal d'orientation fondamentale et stratégique de la Recherche Scientifique et de l'Innovation, initié par le MINRESI tarde à être finalisé. Cela est dû aux difficultés liées au processus d'actualisation du Plan Directeur de la recherche. L'actualisation de ce plan qui devrait permettre d'identifier et de définir les domaines prioritaires de la Recherche pour le développement du pays ; est compromise par la fragilité des Comités de programmes dans les Instituts de recherche. En effets, ces comités du fait du manque des moyens financiers et, surtout de la main d'œuvre en nombre et en qualité ; ont des difficultés à mettre en place des bonnes stratégies de collecte des avis des citoyens. Lesquels avis devraient permettre de mieux prendre en compte la demande sociale dans la mise en œuvre des activités et des projets de recherche. Ils permettraient également d'insérer dans ces projets de recherche, les orientations du gouvernement et la conjoncture économique nationale et internationale dans le but d'inciter les jeunes camerounais à entreprendre dans les activités de création et d'innovation.

De même, une des difficultés auxquelles font face les instituts de recherche sous tutelle du MINRESI pour stimuler l'entrepreneuriat scientifique est due à l'absence d'une structure 
d'intermédiation entre la recherche et le monde des entreprises [CHO 2018]. Selon ce dernier «l'un des obstacles majeurs à la connaissance et à l'exploitation des efforts des chercheurs dans nos pays est l'absence d'une politique de vulgarisation des résultats de la recherche par les chercheurs euxmêmes ou par les institutions pour lesquelles ils travaillent ». Or l'existence d'une telle structure permet de faire le lien entre l'offre des résultats scientifiques et technologiques et leur valorisation industrielle et commerciale.

La réussite de l'entrepreneuriat scientifique dans un pays passe également par la réussite de la mise en place d'un Système National de Recherche Scientifique et d'Innovation (SNRI). Au Cameroun, il est difficile de dire qu'un tel système est effectif, ce pour plusieurs raisons. En effet, d'un point de vue historique, la recherche moderne est introduite au Cameroun par le colonisateur, dans la logique, entre autres, d'un inventaire scientifique du potentiel du Cameroun, conformément à la politique de mise en valeur des colonies. Ici la recherche répond prioritairement aux besoins extérieurs. Ses axes, ses objectifs, son financement, son évaluation et sa coordination dépendent tout aussi de l'extérieur [MBO 04]. A l'indépendance, le jeune Etat hérite alors de certaines de ces différentes structures de recherche. Pendant que d'autres continuent de fonctionner dans le sillage des agences de coopération et de développement des métropoles, et les partenaires bilatéraux et multilatéraux au développement. A sa propre initiative, le pays crée d'abord des écoles et des universités, à l'instar de l'Ecole Nationale Supérieure d'Agronomie (ENSA) ${ }^{13}$, transférée en mai 1977 à Dschang, l'Institut National de Développement Rural (INADER) en 1988 (MINRESI, 2014). A ce jour, plusieurs autres principaux acteurs à l'instar du MINESUP, MINRESI, MINADER ${ }^{14}$ se déploient dans la recherche au Cameroun. A ces ministères s'ajoutent les organisations patronales, les syndicats et les entreprises nationales et internationales ${ }^{15}$, les structures de recherche indépendantes ou personnelles à l'exemple des chercheurs en médecine traditionnelle; les organismes de coopération bilatérale et multilatérale (Agence Universitaire de la Francophonie, Agence Française de Développement, Banque Africaine de Développement...).

Au niveau du MINESUP, deux structures sont en charge de la recherche universitaire. Il y a d'une part, la Direction des Accréditations Universitaires et de la Qualité, via la Sous-Direction de l'Assurance Qualité. Cette dernière est chargé, entre autres, de la participation à l'élaboration des critères de création et de management des laboratoires et des centres de recherche ${ }^{16}$. D'autre part, il y a la Division de la Recherche et de la Coopération universitaires, notamment la Cellule de la Recherche Universitaire. En matière de recherche, cette Division est chargée, entre autres, de l'élaboration du plan directeur de la recherche universitaire et de la promotion de la recherche dans les institutions universitaires. De manière opérationnelle, la Cellule s'occupe, entre autres, de l'élaboration des mesures et dispositions visant la valorisation des résultats de la recherche universitaire en rapport avec les partenaires socioéconomiques ${ }^{17}$. Comme mentionné plus haut, cette diversité de structures nécessite un mécanisme de coordination efficace pour accroitre la performance des différents acteurs. Mais à l'enseignement des faits, le système national éprouve des difficultés de coordination capables de freiner l'entrepreneuriat scientifique dans les universités camerounaises, malgré la mise en place de l'Office National de la Recherche Scientifique et Technique (ONAREST) en 1965.

La réussite de l'entrepreneuriat scientifique nécessite aussi des ressources humaines qualifiées et des infrastructures adaptées. Mais le relatif oubli de la recherche n'a pas favoriser la constitution d'une masse critique d'un capital humain susceptible de piloter la recherche, en tant qu'un objet spécifique, et la mise en place des infrastructures adaptées. Ce relatif oubli de la recherche,

\footnotetext{
${ }^{13}$ Aujourd'hui Faculté d'Agronomie et des Sciences Agricoles (FASA).

${ }^{14}$ Ministère de l'Agriculture et du Développement Rural (MINADER).

${ }^{15}$ Par exemple Cameroon Development Corpation (CDC).

${ }^{16}$ Article 46 du décret $n^{\circ} 2012 / 433$ du 1er octobre 2012 portant organisation du Ministère de l'enseignement supérieur

${ }^{17}$ Articles 64 et 65 du décret n²012/433 du 1er octobre 2012 portant organisation du Ministère de l'enseignement supérieur.
} 
notamment universitaire, se manifeste dès la mise en place de l'enseignement supérieur au Cameroun. Il s'est poursuivi avec l'impératif de faire face à la massification de la population estudiantine et le chômage élevé des diplômés de l'enseignement supérieur. En effet, les multiples structures de recherche qu'hérite le Cameroun lors de son accession à l'indépendance ne sont pas intégrées à l'université. Par conséquent, l'Enseignement Supérieur dès sa naissance se trouve confiné à l'éducation et à la formation. Son but état alors de former les ressources humaines en vue de la prise en main de l'appareil Etatique par les nationaux et le soutien des politiques de développement héritées de la colonisation ou initiées par les autorités du jeune Etat. En outre, en raison de la sensibilité de certaines questions relatives à la gouvernance du jeune Etat, la recherche sur des questions socioéconomiques jugées sensibles est peu admise par les autorités. Il y a alors comme une séparation entre la recherche universitaire et la société. Ce clivage est d'autant plus préjudiciable que le modèle d'université reproduit au Cameroun n'est pas très porté vers les préoccupations socioéconomiques [MBO 04]. Ce mauvais départ a eu, au moins, deux conséquences. D'une part, le MINESUP chargé de fournir une main d'œuvre de qualité aux institutions de recherche sous tutelle du MINRESI a perdu le contrôle de la politique nationale de la recherche. Ce qui a eu un impact négatif sur le système national de la recherche qui se traduit par un manque d'opportunité d'alliage de la recherche à la formation et à l'éducation. D'autre part, cela ne lui a pas permis de se consacrer véritablement à la structuration de la recherche universitaire en interne.

Et, avec les politiques actuelles de massification des effectifs dans les universités qui se sont traduites par une forte croissance de la population estudiantine, sans commune mesure avec les capacités infrastructurelles des universités et les ressources publiques, la priorité dans l'enseignement supérieur s'est orientée vers la recherche des solutions à l'accueil de la population estudiantine. Dans ce contexte de crise économique, les autorités bien qu'en multipliant le nombre des institutions universitaires, des établissements et les filières se sont retrouvées dans un statut quo. Elles se sont en effet, trouvées dans l'obligation de préférer le volet académique, moins exigeant en équipement, en ressources financières; mais aussi peu favorable au développement de l'entrepreneuriat scientifique. Ce qui pose perpétuellement la question de l'inadéquation entre les formations dispensées à l'enseignement supérieur et les réalités socioéconomiques du pays [NGW 2016].

Bien que l'un des palliatifs de premier plan soit la professionnalisation des enseignements, du fait du manque de moyens financiers elle est plutôt portée par l'accroissement qualitatif et quantitatif des établissements technologiques et professionnelles et la modernisation des établissements facultaires classiques. Fondée sur le triptyque «Professionnalisation, employabilité et assurance qualité "), d'après la toile de fond du partenariat université/entreprise, cette situation économique catastrophique conduit toujours à marginaliser le volet recherche. Le tandem université/entreprise n'a surtout exploré jusqu'à présent que la question de la formation, au détriment de celle de la recherche. Les besoins des entreprises en matière de recherche restent mal maitrisés par les universités. De même les différents axes et les résultats de la recherche universitaire demeurent largement méconnus du monde socio-professionnel. Le relatif oubli de la valorisation économique de la recherche n'est pas seulement présent dans la relation universités-entreprises, il serait même la norme de la recherche universitaire au Cameroun. En effet, les chercheurs restent majoritairement tournés vers la valorisation scientifique de la recherche, notamment les publications en vue des changements de grade et le rayonnement scientifique. La valorisation économique est alors marginale. Les relations avec les organismes de protection de la propriété intellectuelle sont insignifiantes. Il n'existe pas de structure capable d'établir un partenariat gagnant-gagnant entre la recherche et le monde des entreprises, bien qu'il existe un plan de mise en place de cette entité. Lequel plan connait d'ailleurs de nombreuses difficultés de financement et de conception.

Et si une telle structure, susceptible de booster le développement socio-économique et culturel du Cameroun existait ; cela ne suffirait pas. Il faudrait aussi l'implication des chercheurs eux-mêmes 
pour impulser la vulgarisation de leurs résultats de recherche auprès des entreprises. En effet, au Cameroun comme partout en Afrique, tel que souligné par [CHO 2018], «piégés dans les méandres de lourdes procédures qui imposent souvent, avant toute sortie dans la presse, une autorisation d'une hiérarchie davantage préoccupée par des considérations politiques plutôt qu'académiques, bon nombre de chercheurs d'Afrique francophone manquent de cette indépendance intellectuelle qui, ailleurs, fait la différence ». Cette nonchalance du chercheur africain conduit à rechercher dans le cadre socioculturel les obstacles au développement de l'entrepreneuriat scientifique dans les instituts de recherche sous la tutelle du MINRESI.

\subsubsection{Les obstacles socioculturels qui enfreignent les objectifs des institutions de recherche}

Plusieurs obstacles socioculturels au développement de l'entrepreneuriat scientifique sont observables dans l'environnement des institutions de recherche au Cameroun. Trois principaux obstacles liés directement aux comportement des camerounais peuvent être soulignés dans le cadre de ce travail.

Le premier obstacle est lié au manque de culture de la science, de la technologie et de l'innovation. Ainsi, comme l'a montré [ETE 2018] « la majorité des Camerounais, par ignorance, ne confère pas à l'innovation la place de choix qu'elle doit occuper dans leurs mœurs. Le progrès social est freiné, le pourcentage des Camerounais qui s'intéresse la recherche et à ses résultats est faible». D'après ce dernier, ce désintérêt vis-à-vis de la recherche scientifique, la création et l'innovation est également appréhendé par la méconnaissance de l'existence du MINRESI qui est en charge de la promotion et de l'appui à l'innovation. Ses services, pourtant ouverts au grand public, sont très peu sollicités par les camerounais. Ce qui traduit une absence de prise de conscience sur des questions relatives à l'importance de la recherche scientifique et de l'innovation; non seulement pour la réduction de la pauvreté au niveau individuel, mais aussi pour le développement économique du pays. Par conséquent, en s'inscrivant dans la même logique que [ETE 2018], on peut affirmer que du fait de l'insuffisance des savoir-faire, faute d'esprit de recherche, de créativité et d'innovation, l'inertie et la routine sont devenues les caractéristiques principales des camerounais. Ce qui est observable dans leurs activités au quotidien. Pourtant, pour améliorer impulser le développement économique de leur pays les citoyens doivent s'appuyer sur la recherche et l'innovation technique. Pour ce faire, cela nécessite qu'ils s'intéressent aux formations scientifiques de haut niveau, d'ingénierie et de technologie. En effet, le développement de l'entrepreneuriat scientifique nécessite certes la capacité à innover qui est possible non seulement à travers l'esprit de créativité, mais aussi à partir d'une formation dans le domaine de la conception, la production et la modernisation. Ce qui n'est pas le cas au Cameroun où plusieurs personnes recensées, qualifiées d'innovateurs n'ont pas de formation académique, même si pour certains auteurs [CHO 2018], « ce n'est pas la compétence ni la créativité » qui manquent aux chercheurs et inventeurs camerounais. Cette contradiction amène à observer les facteurs socioculturels tels que les choix de formation académique, comme élément essentiel d'explication du manque de développement de l'entrepreneuriat scientifique au Cameroun.

Le second obstacle est relatif à l'existence d'une forte proportion de personnes à formation généraliste. En effet, le système éducatif camerounais est bondé de plusieurs jeunes qui choisissent plus à fréquenter l'enseignement général. Ce choix est lié à la mentalité des camerounais qui préfèrent exercer des tâches administratives. Le tableau 5 montre qu'une importante partie de la population en âge de travailler $(72,6 \%)$, définie au sens du Bureau international du travail (BIT) ${ }^{18}$, n'a pas reçu de formation professionnelle. Il ressort aussi de ce tableau que $70,2 \%$ de personnes occupées par une activité économique, $57,7 \%$ de chômeurs et $82 \%$ d'inactifs n'ont pas reçu de formation professionnelle.

\footnotetext{
${ }^{18}$ Il s'agit des personnes ayant au moins 15 ans.
} 


\begin{tabular}{|c|c|c|c|c|}
\hline Statut de formation professionnelle & Occupés & $\begin{array}{c}\text { Chômeurs au sens } \\
\text { élargi du BIT }\end{array}$ & Inactifs & Ensemble \\
\hline Formation professionnelle non reçue & 70,2 & 57,7 & 82 & 72,6 \\
Formation professionnelle reçue & 29,8 & 42,3 & 18 & 27,4 \\
Total & $\mathbf{1 0 0}$ & $\mathbf{1 0 0}$ & 100 & $\mathbf{1 0 0}$ \\
\hline
\end{tabular}

Source : INS, 2e Enquête sur l'Emploi et le Secteur Informel, 2010

Tableau 5. Proportion de la population en âge de travailler suivant leur situation d'activité et statut de formation professionnelle

Ce constat découlant du tableau 5 signifie que le marché de travail au Cameroun compte, majoritairement, des personnes à formation généraliste. D'ailleurs le graphique 5 ci-dessous le prouve. Sur ce graphique il peut être constaté que le marché du travail camerounais regorge en majorité des personnes non scolarisées et celles de niveau d'étude du primaire et secondaire général. La plupart d'entre elles qui ont les niveaux d'étude du secondaire général sont au chômage. On a respectivement $30 \%$ et $14 \%$ pour le $1^{\text {er }}$ cycle et second cycle contre $20 \%$ et $7 \%$ d'occupés. En dehors des faibles effectifs qui sortent des écoles techniques et dont la moitié est occupée, très peu de personnes sorties de l'enseignement supérieur sont occupées. Parmi celles qui sont occupées, moins de 5\% sont diplômés de l'enseignement supérieur et 15\% des chômeurs sont diplômés de cet ordre d'enseignement.

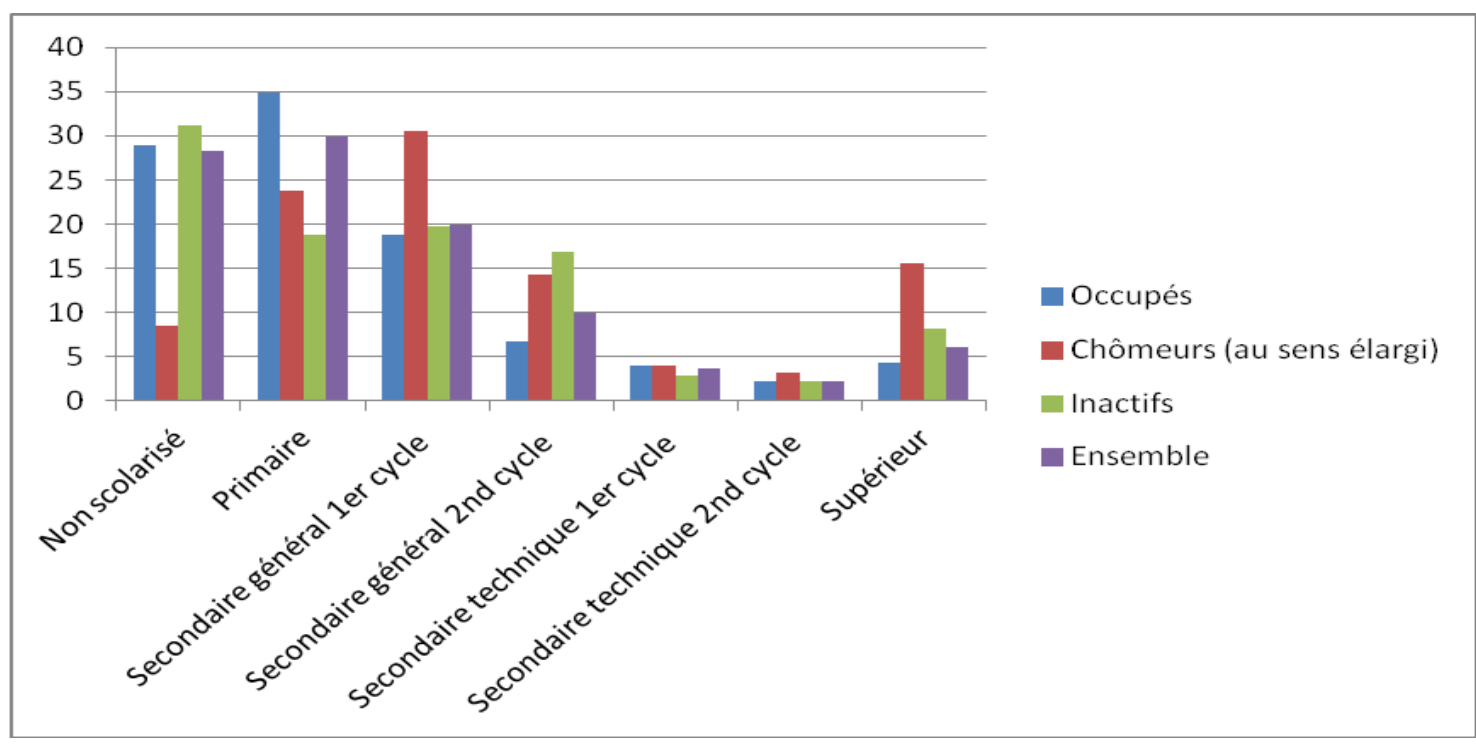

Source : INS, 2e Enquête sur l'Emploi et le Secteur Informel, 2010

Graphique 2. Répartition (en \%) de la population en âge de travailler suivant leur situation d'activité et le niveau d'instruction

Parmi les personnes occupées, $28 \%$ sont non scolarisées contre $8 \%$ qui sont des chômeurs. Et $35 \%$ ont le niveau d'étude du primaire contre $18 \%$ de chômeurs. Il faut dire qu'en réalité ce sont des gens qui exercent dans le secteur primaire et principalement dans une agriculture rudimentaire $(45,3 \%)$ et dans les activités ménagères $(24,4 \%)$ et de gardiennage ${ }^{19}$. Cette triste réalité de l'économie camerounaise est davantage amplifiée par l'incapacité de la main d'œuvre active à entreprendre. On y assiste à une préférence pour l'emploi salarié. 
Le troisième obstacle est lié à une forte préférence pour l'emploi salarié. Ainsi, souvent même s'ils ont reçu une formation scientifique, d'ingénieur ou en technologie, une importante partie de ces camerounais préfèrent des emplois salariés au lieu de créer une entreprise et de s'auto employer ou d'entreprendre dans la recherche scientifique pour soit innover ou créer de nouveaux produits. Sur le graphique 6, on peut constater que dans l'ensemble les chômeurs ont une préférence pour l'emploi salarié. Le pourcentage des personnes de cet avis s'élève à plus de $50 \%$.

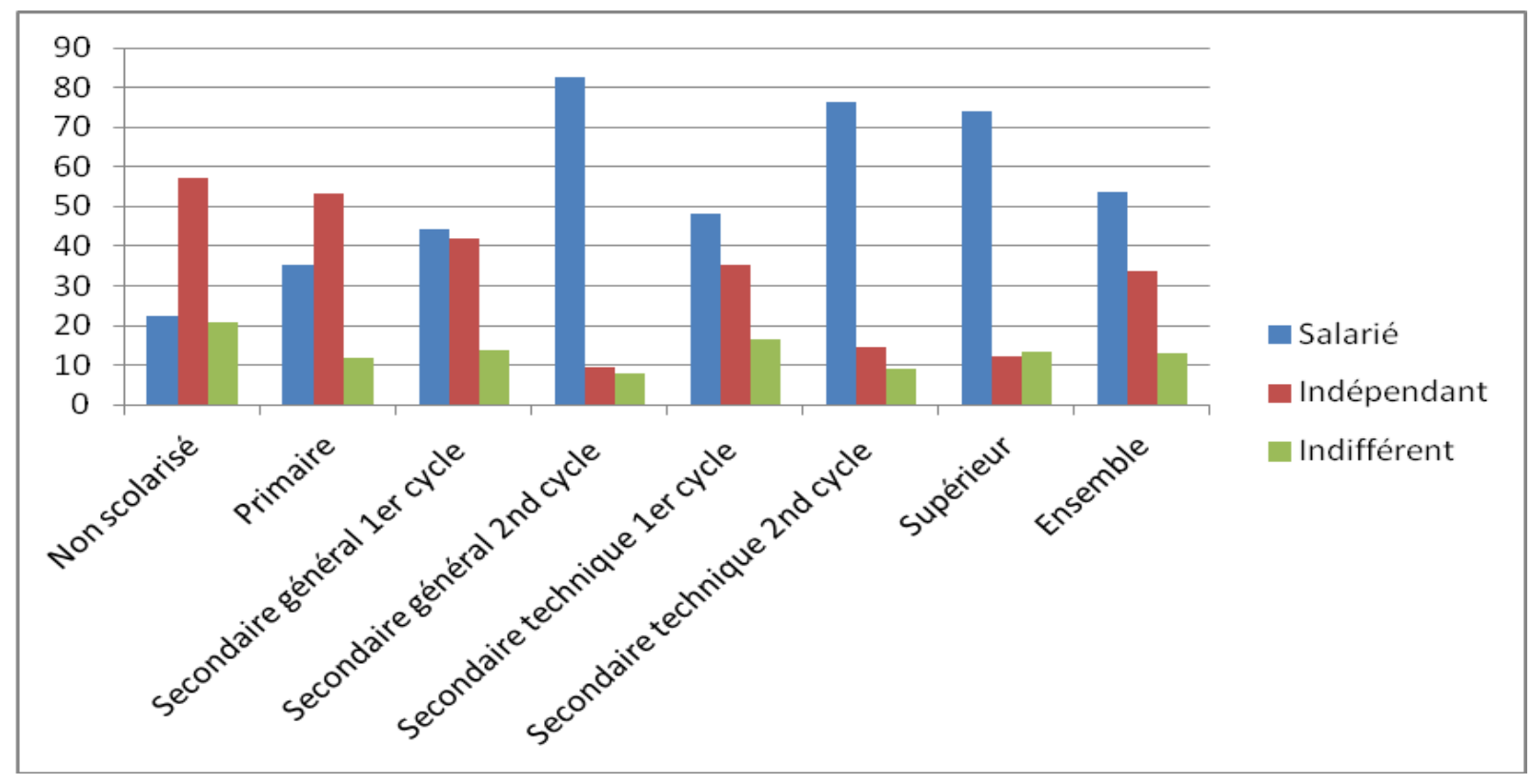

Source : INS, 2e Enquête sur l'Emploi et le Secteur Informel, 2010

Graphique 3. Proportions des chômeurs par niveau d'éducation et type d'emploi recherché

Toutefois, cette préférence est plus élevée chez les diplômés des seconds cycles général et technique et ceux des universités. En effet, parmi les sortants de ces différents ordres d'enseignement, respectivement $81 \%, 77 \%$ et $72 \%$ préfèrent l'emploi salarié. Par contre une importante partie des personnes non scolarisées et celles qui ont abandonnées les études au niveau primaire préfèrent travailler à leur propre compte. Cependant leur nombre reste inférieur à ceux qui préfèrent l'emploi salarié. Ce constat pousse à affirmer que les curricula mis à la disposition des étudiants camerounais ne permettent pas de développer en eux l'esprit entrepreneurial. Les raisons de l'incapacité à développer l'entrepreneuriat en général et, particulièrement l'entrepreneuriat scientifique sont également recensées dans le contexte économique de ce pays.

\section{Un contexte économique qui freine les objectifs d'entrepreneuriat scientifique}

Dans le but de parvenir à un développement économique et social soutenu, le gouvernement camerounais a mis sur pied, en 2010, un Document de Stratégie pour la Croissance et l'Emploi (DSCE). Il s'agit d'un document qui comporte des mesures de politiques publiques à mettre en œuvre. Malgré la mise sur pied de ce dispositif, la situation économique ne s'améliore pas substantiellement. Ce qui a un impact sur la formation de la jeunesse, le marché de travail et l'entrepreneuriat tant au niveau global qu'au niveau des universités; surtout lorsqu'il s'agit de prendre en compte l'évolution démographique.

\subsection{Contexte économique et financement des activités de recherche scientifique}

Deux points sont soulignés ici : l'analyse de la situation économique du Cameroun et la difficulté de l'économie à financer l'entrepreneuriat scientifique. 


\subsubsection{Analyse de la situation économique et financière du Cameroun}

Le Cameroun comme de nombreux pays africains connait de nombreuses difficultés tant sur le plan économique que financier. Le graphique 2 , montre que ce pays fait face à de difficultés d'ordre financière.

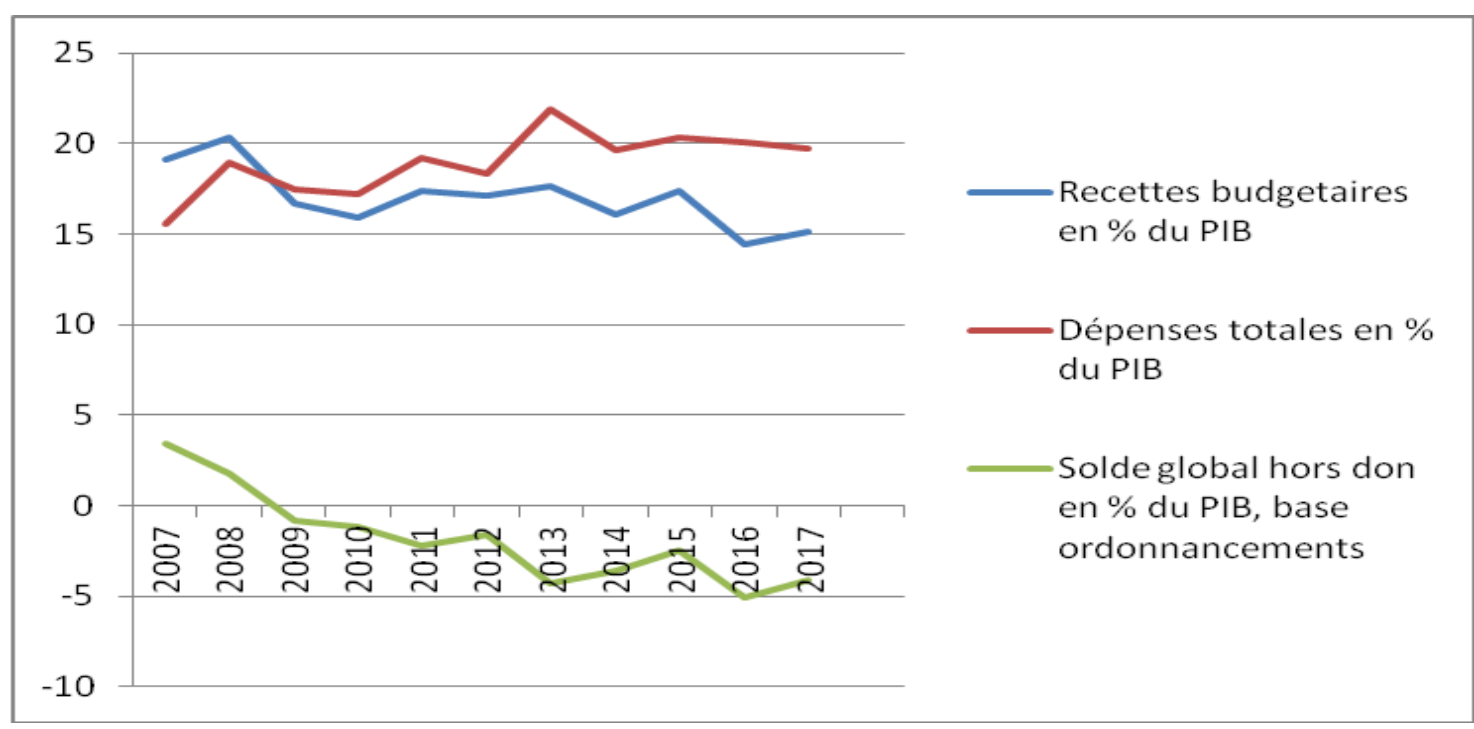

Source : Données du Ministère des Finances/ Direction des Affaires Economiques

Graphique 4. Evolution (en \% du PIB) des recettes, dépenses et soldes budgétaires entre 2007-2017

Entre les années 2009 et 2017, les recettes budgétaires en pourcentage du PIB enregistrées sont inférieures aux dépenses totales de l'Etat impliquant un solde budgétaire négatif. Cette incapacité à collecter suffisamment les recettes budgétaires marque la difficulté habituelle du Cameroun à pouvoir financer normalement toutes ses activités régaliennes et particulièrement les activités de recherche et de l'innovation comme. Ce qui constitue un blocage au développement de l'entrepreneuriat scientifique.

\subsubsection{Des difficultés à financer la recherche et l'innovation technologique}

Plusieurs facteurs expliquent l'incapacité de l'Etat du Cameroun à collecter suffisamment les recettes budgétaires afin de financer les activités de recherche et de l'innovation technologique. Au moins quatre raisons peuvent être évoquées ici. La première raison est attribuable au caractère dual de l'économie camerounaise. Il s'agit d'une économie caractérisée d'un côté par un secteur formel étroit, intégré dans les marchés mondiaux et régionaux. Dans ce secteur l'Etat parvient à collecter les taxes, contrôler la qualité et les prix des produits, instaurer l'ordre public et la sécurité sociale etc. De l'autre côté, un secteur informel puissant qui permet simplement à la plupart de la population d'ailleurs pauvre de survivre de leurs activités précaires et où l'Etat est incapable de collecter les recettes fiscales. Ce secteur à faible productivité occupe près de $90 \%$ des travailleurs et la plupart des personnes en âge de travailler ; sorties des écoles et universités s'y insèrent (EESI-2, 2010) ${ }^{20}$.

La deuxième raison est la place accordée au institutions de recherche scientifique et technologique par les pouvoirs publics. En effet, les budgets alloués au fonctionnement de ces institutions sont faibles même si dans l'ensemble les autres ordres d'enseignement (Education de base et Enseignement secondaire) reçoivent des montants très importants. Le tableau 6 montre qu'au niveau de l'enseignement supérieur le budget alloué à cet ordre d'enseignement est passé de 51,9 milliards en 2015 à 47,6 milliards en 2016 (Loi de finances, 2016). Ce qui constitue un frein pour

\footnotetext{
${ }^{20}$ Deuxième Enquête sur l'Emploi et le Secteur Informel. C'est un secteur à faible productivité qui occupe près de $90 \%$ des travailleurs en 2010.
} 
cet ordre d'enseignement à atteindre ses objectifs stratégiques que sont la professionnalisation des enseignements, la modernisation des établissements et facultés et le développement de la recherche et l'innovation dans les universités. De plus, même si parfois le budget MINESUP est revu à la hausse (67,6 milliards en 2017), la part qui lui est accordée dans le budget de l'Etat n'atteint jamais $2 \%$. Cette situation est plus critique dans le cas du MINRESI.

\begin{tabular}{|l|c|c|c|c|c|c|c|}
\hline Année & $\mathbf{2 0 1 5}$ & $\mathbf{2 0 1 6}$ & $\mathbf{2 0 1 7}$ & $\mathbf{2 0 1 8}$ & $\mathbf{2 0 1 9}$ & $\mathbf{2 0 2 0}$ & $\mathbf{2 0 2 1}$ \\
\hline Budget de l'Etat & 3746000 & 4234000 & 4373000 & 4689500 & 4850500 & 4951711 & 4670000 \\
\hline Budget Secteur Education & 521028 & 527868 & 651429 & 673494 & 711032 & 760687 & 653336 \\
\hline Budget MINESUP & $\mathbf{5 1 9 4 4}$ & $\mathbf{4 7 6 4 4}$ & $\mathbf{6 7 6 5 4}$ & $\mathbf{6 1 4 0 1}$ & $\mathbf{5 5 9 5 2}$ & $\mathbf{6 5 2 2 8}$ & $\mathbf{6 5 1 4 8}$ \\
\hline Budget MINRESI & $\mathbf{1 3 8 4 7}$ & $\mathbf{1 2 ~ 8 3 7}$ & $\mathbf{8 5 8 4}$ & $\mathbf{1 0 3 0 0}$ & $\mathbf{1 1 9 1 6}$ & $\mathbf{1 0 ~ 9 0 2}$ & $\mathbf{8 6 9 1}$ \\
\hline $\begin{array}{l}\text { Part du budget du secteur } \\
\text { Education (\%) }\end{array}$ & $13,91 \%$ & $12,47 \%$ & $14,90 \%$ & $14,36 \%$ & $14,66 \%$ & $15,36 \%$ & $13,99 \%$ \\
\hline $\begin{array}{l}\text { Part du Budget du } \\
\text { MINESUP (\%) }\end{array}$ & $\mathbf{1 , 3 9 \%}$ & $\mathbf{1 , 1 3 \%}$ & $\mathbf{1 , 5 5 \%}$ & $\mathbf{1 , 3 1 \%}$ & $\mathbf{1 , 1 5 \%}$ & $\mathbf{1 , 3 2 \%}$ & $\mathbf{1 , 4 0 \%}$ \\
\hline $\begin{array}{l}\text { Part du Budget du } \\
\text { MINRESI (\%) }\end{array}$ & $\mathbf{0 , 3 6 \%}$ & $\mathbf{0 , 3 0 \%}$ & $\mathbf{0 , 2 0 \%}$ & $\mathbf{0 , 2 1 \%}$ & $\mathbf{0 , 2 5 \%}$ & $\mathbf{0 , 2 2 \%}$ & $\mathbf{0 , 1 9 \%}$ \\
\hline
\end{tabular}

Source : Lois de finance : 2015 à 2021

Tableau 6. Part du MINESUP et MINRESI dans le budget de l'Etat (en milliards de FCFA)

La part accordée au MINRESI dans le Budget de l'Etat n'a jamais atteint 0,4\% entre les années 2015 et 2021. Que soit dans le cas du MINESUP ou du MINRESI, malgré l'augmentation du budget de l'Etat, il arrive parfois que leurs parts baissent. Cette négligence par les pouvoirs publics camerounais semble constituer un frein au développement de l'entrepreneuriat scientifique dans ce pays.

La troisième raison est relative à l'insuffisance en information économique, malgré l'existence d'une structure de facilitation de l'emploi jeune, dénommée Fonds National de l'Emploi (FNE). Le Ministère de l'Economie à travers l'Institut National de la Statistique et le FNE, dont l'une des principales missions est le financement des projets de création d'entreprise, ont identifié en 2014 les contraintes auxquels les jeunes camerounais sortant des écoles de formation tant primaires, secondaires que supérieures font face. Parmi elles, il y'a la question de l'insuffisance en information économique dont l'intérêt est de faciliter l'accès à une idée de projet, aux choix technologiques, aux sources de financement, aux circuits d'approvisionnement en matières premières et de distribution des biens produits. Il y a également une faible capacité technique des promoteurs pour l'assistance et la conduite de l'élaboration de plans d'affaires et la confection d'un dossier de projet bancable. A ceci, s'ajoute un déficit en suivi/encadrement pendant les premières années de démarrage des entreprises.

Récemment, en 2016, l'INS a identifié d'autres obstacles qui ont trait au niveau de fiscalité, à l'ampleur de la corruption, à la difficulté d'accès au crédit, aux formalités administratives qui sont rigides, à la concurrence déloyale des grandes entreprises, la mauvaise qualité des infrastructures, au coût de financements perçus par les promoteurs d'entreprises, à la mauvaise qualité de l'énergie électrique, etc... A côté de ces difficultés il faut aussi relever celles relatives au recouvrement de créances du fait de la mauvaise foi des entrepreneurs, la faiblesse du ratio des projets financés par les programmes nationaux par rapport à la demande et le niveau faible des montants à accorder [NSA 2013]. Elles montrent que l'objectif gouvernemental de mise en place d'un Système National de Recherche Scientifique est difficile à atteindre. Par ailleurs, cette situation économique difficile est à l'origine de nombreuses conséquences sociales néfastes qui perdurent et inhibent davantage l'ambition de développement de l'entrepreneuriat scientifique au Cameroun. 


\subsection{Les conséquences d'un contexte économique difficile sur l'objectif l'entrepreneuriat scientifique}

Ces conséquences peuvent être évoquées à plusieurs niveaux : 1) la relation entre la jeunesse entreprenante et les pouvoirs publiques, 2) l'ampleur du secteur informel, 3) la détérioration du pouvoir d'achat et 4) la fragilité du marché du travail.

\subsubsection{Une mauvaise relation entre la jeunesse et les pouvoirs publics}

Face à cette situation économique difficile, l'Etat camerounais a mis sur pieds des instruments pour résoudre le problème d'emploi des jeunes. Parmi ceux-ci il y a le Fonds National de l'Emploi (FNE), l'Observatoire National de l'Emploi et de la Formation Professionnelle (ONEFOP), le Projet d'Appui au Développement des Emplois Ruraux (PADER) et les Centres de Formalités de Création d'entreprise (CFCE). Ces institutions ont pour but d'encourager l'entrepreneuriat des jeunes. Cependant, elles se trouvent confrontées à des difficultés pour soutenir l'entrepreneuriat. Par conséquent, l'incapacité à accéder aux financements publiques et la pression fiscale conduisent à une mauvaise atmosphère entre les jeunes entrepreneurs et les dirigeants. Selon les jeunes, l'existence des structures de soutien mises à leur disposition ne parviennent pas à satisfaire leur demande. Par exemple, d'après le précédent rapport thématique, environ $46 \%$ des entrepreneurs trouvent que leurs relations avec les pouvoirs publics sont « mauvaises » contre 32,8\% d'entre eux qui estiment qu'elles sont « bonnes ». Ce qui démontre une situation désagréable de l'environnement des affaires. D'où, l'émergence d'un marché de travail fragile, dominé par l'amateurisme.

\subsubsection{Le développement d'une économie informelle}

La situation économique difficile engendre une prolifération des activités informelles. Ces activités précaires et informelles réalisées par la grande majorité des camerounais ne permettent pas d'améliorer leurs conditions de vie. En effet, la plupart de ces activités ne sont pas comptabilisées dans le calcul du PIB. Et, face à un taux de croissance démographique constant de plus de 2,5\%, les différentes cohortes sortant du système éducatif, convergent pour la plupart vers ces activités amplifiant ainsi les effectifs d'emplois créés dans ce secteur. Ce qui réduit substantiellement le taux de croissance du PIB réel par tête comme le montre le graphique 3.

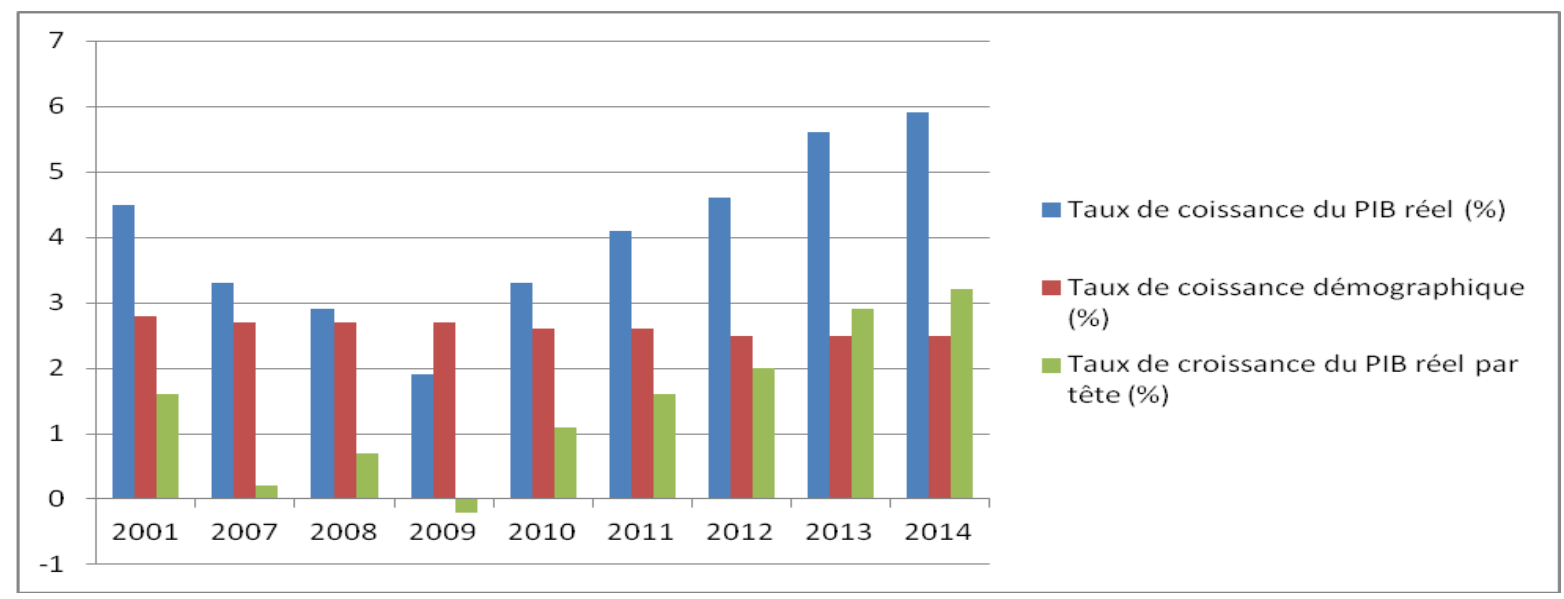

Sources : - Ministère des Finances/ Direction des Affaires Economiques, -Bureau Central du Recensement et des Etudes de Population $/ 3^{e}$ RGPH

Graphique 3. Evolution (en \%) des taux de croissance du PIB réel et démographique entre 2001-2014

Parmi les personnes qui travaillent dans le secteur informel, une importante partie exerce dans les activités telles que l'agriculture, l'élevage et la pêche. Il s'agit d'une agriculture rudimentaire, non mécanisée et dont la productivité est limitée par le changement climatique et l'incapacité à supporter 
les charges de production élevées par les agriculteurs. De nos jours, cette activité est progressivement délaissée par les jeunes camerounais sortis des écoles qui préfèrent exercer dans l'informel [INS 2011] où ils écoulent essentiellement les biens manufacturés de seconde main, la friperie et les produits alimentaires importés. Du coup, il est observé chaque année une hausse des prix des produits alimentaires, souvent plus que la hausse des prix des autres biens comme le montre le graphique 4 .

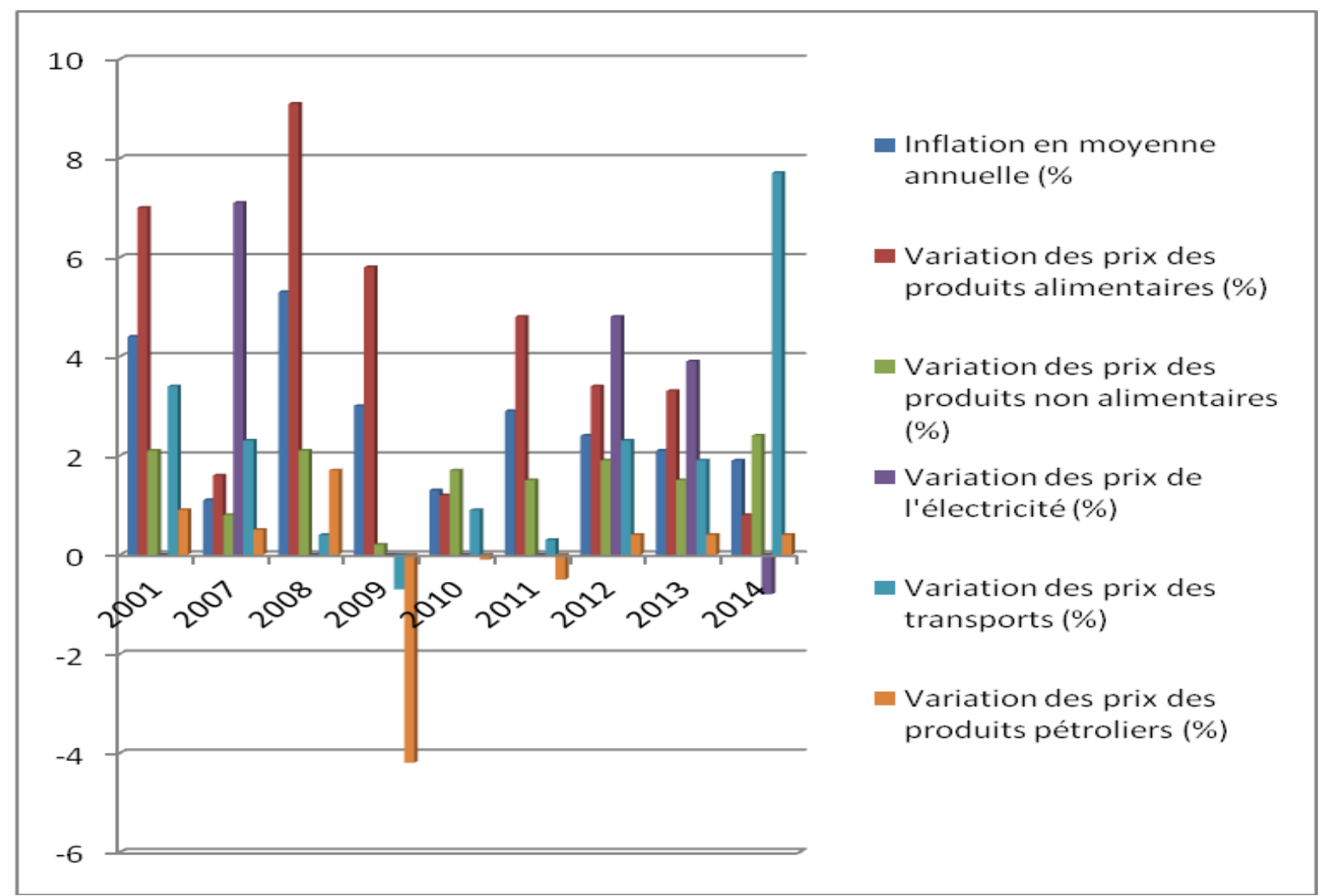

Source : Ministère des Finances/ Direction des Affaires Economiques

Graphique 4. Evolution (en \%) du taux d'inflation par rapport à la variation des autres types de bien

\subsubsection{Un marché de travail fragile, incapable à absorber la main-d'œuvre et à stimuler l'entrepreneuriat scientifique}

Le marché du travail au Cameroun est fragile du fait de son inefficacité à absorber la main d'œuvre qui sort des écoles de formation. C'est un marché où le taux de sous-emploi est très critique et une importante partie de la main d'œuvre n'a pas reçue de formation professionnelle. Sur ce marché le taux de création d'emplois est très faible comparativement aux effectifs sortis des écoles de formation. De plus, le volume des biens manufacturés importé est plus important que la production domestique.

\subsubsection{Un taux de sous-emploi élevé}

Le tableau 7 démontre que le marché du travail au Cameroun est caractérisé par un sous-emploi très élevé. En effet, en 2010 le taux de sous-emploi global au sens du BIT s'élevait à 70,6\%. Ce taux est moins élevé au niveau des employés ayant le niveau du supérieur (44,3\%) comparativement à tous les autres ordres d'instruction. Ce résultat pousse à croire que les sortants des universités sont mieux employés que les autres diplômés. Ce qui semble ne pas être juste dans la mesure où le taux de chômage des personnes en situation de sous-emploi global est largement supérieur au taux de chômage des diplômés des autres niveaux d'éducation. 


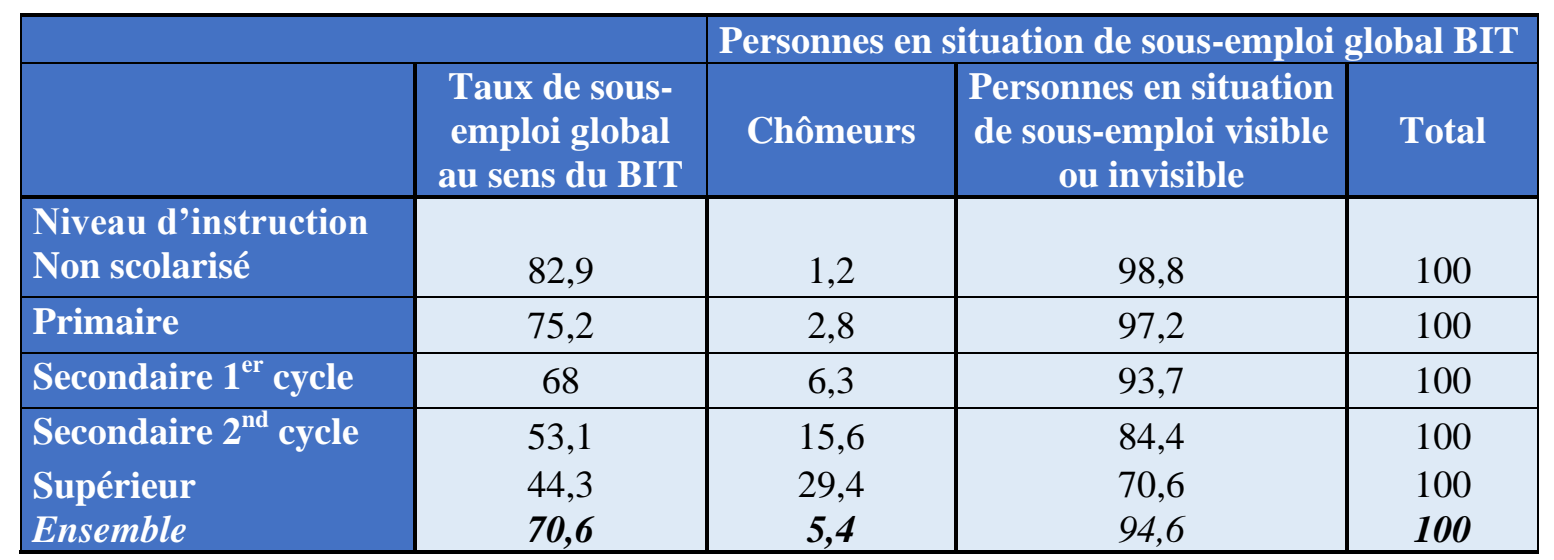

Source: INS, 2e Enquête sur l'Emploi et le Secteur Informel, 2010

Tableau 7. Taux de sous-emploi global selon quelques caractéristiques contextuelles

A partir de ce tableau 7, il peut être conclu que l'économie du Cameroun est très fragile et incapable non seulement à utiliser mais davantage à absorber la plus grande partie de la main d'œuvre existante et surtout celle qui est formée. En effet la main d'œuvre existante est presqu'entièrement oisive. Cette affirmation peut être étayée à partir du point suivant.

\subsubsection{Une économie incapable à absorber la main d'œuvre qualifiée}

Le tableau 8 présente les niveaux de créations nettes d'emploi des différents types structures de l'économie entre les dates 2011 et 2013. A l'analyse de ce tableau, il est évident de conclure que le secteur productif au Cameroun ne crée pas suffisamment d'emplois.

\begin{tabular}{|c|c|c|c|c|c|}
\hline & & & & 2012 & 2013 \\
\hline & 2011 & 2012 & 2013 & $\begin{array}{l}\text { Créations } \\
\text { nettes }\end{array}$ & $\begin{array}{c}\text { Créations } \\
\text { nettes }\end{array}$ \\
\hline Secteur productif moderne & 544700 & 612381 & 653126 & 67681 & 40745 \\
\hline Etablissement de micro finance et GICS & 6066 & 6176 & 6714 & 110 & 538 \\
\hline Secteur Public & & & & & \\
\hline -Fonction Publique et forces de défense et sécurité & 273110 & 305153 & 329000 & 32043 & 23847 \\
\hline -Etablissement Publique Administratifs & 220777 & 249899 & 264000 & 29112 & 14101 \\
\hline -Collectivités Territoriales Décentralisées & 39400 & 41801 & 42111 & 2401 & 310 \\
\hline -Grands projets du gouvernement & 12933 & 13453 & 14423 & 520 & 970 \\
\hline Ensemble & 815042 & 914741 & 979406 & 99699 & 64665 \\
\hline
\end{tabular}

Source : MINFI, MINFOPRA, INS/enquête emploi 2013

Tableau 8. Evolution des emplois par secteur productif et public entre 2011 à 2013

En se référant aux tableaux 1 et 3, on constate que les effectifs des étudiants fréquentant les universités publiques et privées durant les années académiques 2011/2012 et 2012/2013 sont respectivement de 252376 et 222604 et le nombre de diplômés sortant de ces universités s'élèvent à 45775 pour l'année 2012. Si l'on ajoute à cet effectif des diplômés d'universités, les $123386^{21}$ sortants des centres de formation des ministères de la formation professionnelle, de l'agriculture, de l'élevage, des enseignements secondaires, de la jeunesse et des sports, il est aisé de constater que le nombre d'emplois (99 699) créés par l'économie est largement inférieur aux effectifs des diplômés pour une année. Cette réalité est plus mauvaise surtout s'il faut prendre en compte les effectifs sortis des établissements secondaires d'enseignement technique. Malgré cette situation catastrophique dans laquelle se trouve l'économie camerounaise ; qui bloque son processus de développement de l'entrepreneuriat culturel, quelques cas de succès peuvent être relevés. Ces cas de succès

\footnotetext{
${ }^{21}$ Sources de Calcul : INS-Annuaire statistique-2013, DEFACC/Ministère de l'Agriculture et Ministère de l'élevage. 
proviennent des rares cas de partenariats que certains établissements des universités ou d'instituts de recherche ont tissé soit avec les entreprises soit avec les universités étrangères.

\section{Des partenariats Universités-entreprises et coopérations universitaires internationales}

$\mathrm{Au}$ Cameroun, la relation des universités avec les entreprises reste très faible bien que les résultats des assises sous régionales de $2008^{22}$ mettent un accent particulier à cette question. Ces assises recommandent comme solution idoine la participation des professionnels aux enseignements. Se fondant sur les recommandations de ces assisses, un cadre juridique a été élaboré pour rendre crédible ce type de partenariat. Il a été question de créer par un décret ${ }^{23}$ un diplôme d'habilitation à dispenser des enseignements techniques et professionnels (DHDTP) dans le but d'inciter les professionnels à enseigner à l'université et donner une crédibilité aux enseignements dispensés. Depuis la publication de ces recommandations, plusieurs établissements d'enseignement supérieur à l'instar de l'Ecole Supérieure des Sciences Economiques et Commerciales (ESSEC) de Douala, les Instituts Universitaires de Technologies (IUT) de Douala, N'Gaoundéré et Dschang accueillent des enseignants professionnels et encouragent les enseignants permanents à fréquenter le monde de l'entreprise et des consultations. Cependant, l'on peut constater que ces efforts de professionnalisation font face à des obstacles. Par exemple, au niveau universitaire, on peut observer la difficulté psychologique qui anime les enseignants permanents à accepter cette réforme. En effet, ceux-ci craignent de voir leur compétence scientifique se détériorer, car partir des compétences à transmettre pour le marché du travail ne va pas de soi [DEL 2010]. Pourtant, comme le montre ces derniers la majorité des enseignants camerounais ont effectué leurs études à l'étranger. Et c'est même à l'étranger qu'ils y ont fait leurs recherches. Cette situation amène à s'interroger dans la mesure où l'état de la recherche est fragile et que le monde économique fait face à une pénurie de jeunes talents qui sont en mesure de dynamiser le pays à la recherche de nouveaux savoir-faire.

En revanche, de nombreux établissements universitaires continuent à entretenir des coopérations avec des universités étrangères surtout occidentales. Ces coopérations et même les plus anciennes intègrent aujourd'hui dans leur démarche la notion de la professionnalisation des enseignements. Parmi elles, l'on peut qualifier les suivantes comme les plus importantes qui visent cette professionnalisation : - l'Institut Régional de Coopération Développement (IRCOD) qui vient en appui au développement des établissements technologiques de l'enseignement supérieur au Cameroun depuis environ 30 ans. - la Fondation Internationale pour la Science (FIS) qui contribue au renforcement des capacités scientifiques des pays en développement dans les domaines relatifs à la gestion, la conservation et le renouvellement des ressources naturelles. - Agropolis International, qui effectue une mission d'assistance technique sur le projet de création d'incubateurs d'entreprises au sein des établissements d'Enseignements Supérieurs Camerounais depuis 2008. Ce projet est financé par la coopération française. A présent les établissements bénéficiaires de cette expertise sont les universités de Douala, Yaoundé I, N'gaoundéré et l'Ecole Supérieure Polytechnique de Yaoundé.

$\mathrm{Du}$ fait de ces partenariats, des cas rares de succès relatifs à la mise sur pieds d'universités entrepreneuriales peuvent être relevés. En effet, la coopération entre IUT de Douala et IRCOD a permis aux apprenants de l'IUT de fabriquer des pièces métallurgiques qui sont demandés par de nombreuses entreprises. La coopération bénéficiant de l'expertise d'Agropilis International a permis de doter les enseignants de la Faculté du Génie Industriel (FGI) de l'université de Douala d'un cadre adéquat de travail et de professionnaliser les enseignements. Aujourd'hui, la FGI offres différentes

\footnotetext{
${ }^{22}$ Ministère de l'Enseignement Supérieur, Atelier sous régional sur les programmes de formation de la composante technologique et professionnelle, Douala- CAMEROUN du 7 au 11 Juillet 2008.

${ }^{23}$ Le décret 10-397 a été pris le 16 novembre 2010, fixant le cadre et l'organisation des habilitations à dispenser des enseignements professionnels.
} 
formations dont les ressortissants sont réquisitionnés par les entreprises chinoises dès la $3^{\text {ème }}$ année de licence d'ingénieur. L'Ecole Nationale Supérieure Polytechnique (ENSP) de Yaoundé I, en plus des soutiens d'Agropolis et d'IRCOD, reçoit des nombreux projets mondiaux, européens ou africains des fonds qui lui permettent de professionnaliser ses enseignements (cti, 2017) ${ }^{24}$. Ces coopérations efficaces et permanentes lui permettent aussi de multiplier ses relations avec les entreprises nationales qui par ricochet offrent des stages aux apprenants. Ce qui permet d'offrir aux étudiants un enseignement connecté à la réalité et un accès rapide au marché du travail. Ces coopérations ont aussi permis de mettre sur pied la Junior Entreprise par le biais de laquelle l'école parachève la formation des étudiants et les mettent à la disposition des entreprises. Celle-ci offre également des services aux tiers ; ce qui permet à l'ENSP d'augmenter son budget. Si certains de ces partenariats ont porté des fruits, il n'en demeure pas moins que ces efforts sont estompés par la situation économique du Cameroun.

\section{Conclusion}

Le présent article avait pour ambition de passer en revue les différents éléments structurels, institutionnels et organisationnels qui inhibent l'émergence d'un cadre susceptible de favoriser l'entrepreneuriat scientifique au Cameroun. Pour y parvenir, il a semblé important de mettre l'accent, d'une part, sur le contexte socio- institutionnel des universités, et d'autre part, sur le contexte économique du Cameroun. Mais au préalable il a fallu passer en revue les travaux traitant des effets de l'environnement social, institutionnel et économique des établissements d'enseignement supérieur et de recherche sur la capacité d'un pays à développer l'entrepreneuriat scientifique. Cela a été possible grâce aux sources documentaires et aux données statistiques à l'Institution National de la Statistique (INS), et certains ministères.

Le constat qui se dégage relativement au contexte socio-institutionnel, est que contrairement aux pays industriels tels que le Canada, les Etats Unis [MAT 2015] et certains pays d'Afrique du nord à l'instar du Maroc [BEL 2018] qui ont pu mettre en place des préalables qui ont favorisé un exploit en termes d'entrepreneuriat scientifique, l'environnement de la recherche scientifique au Cameroun fait face à un mécanisme national de coordination inefficace. Ce qui rend impossible toute tentative de déploiement au sein des universités des dispositifs d'incitation à l'entrepreneuriat scientifique, ni de valorisation des résultats de recherche. Le cadre réglementaire des activités de recherche est dispersé entre plusieurs institutions, engendrant de ce fait des freins d'ordre managérial. Il y a un manque de culture de la science, de la technologie et de l'innovation. Très peu d'étudiants fréquentent des filières d'ingénierie, d'industrie de transformation et de production. Ils ont une forte préférence pour l'emploi salarié. Sur le plan infrastructurel, il y a un manque d'infrastructures qui génère de mauvais indicateurs formation à savoir de mauvais ratios d'encadrement suite à une croissance exponentielle des effectifs d'étudiants au fil des ans. A cela s'ajoutent des difficultés liées au processus d'actualisation du Plan Directeur de la recherche du fait du manque des moyens financiers et, surtout de la main d'œuvre en nombre et en qualité. Il y a également l'absence d'une structure d'intermédiation entre la recherche et le monde des entreprises, c'est-à-dire l'absence d'un Système National de Recherche Scientifique et d'Innovation. S'agissant du contexte économique, il faut noter les difficultés à financer la recherche et l'innovation technologique proviennent de l'incapacité de l'Etat du Cameroun à collecter suffisamment les recettes budgétaires. Les budgets alloués au fonctionnement de ces institutions sont faibles même si dans l'ensemble les autres ordres d'enseignement (Education de base et Enseignement secondaire) reçoivent des montants très importants.

Comme solution pour améliorer le cadre socio-institutionnel et impulser l'entrepreneuriat scientifique, le gouvernement devrait actualiser le Plan Directeur de la recherche existant, dans le

\footnotetext{
${ }^{24}$ Commission des titres d'ingénieur (cti), Rapport de mission d'audit à L'ENSP.
} 
but d'identifier et de circonscrire les domaines prioritaires de la Recherche pour le développement du pays. Il devrait élaborer des stratégies visant à vulgariser auprès des populations, y compris les jeunes et les femmes, la culture de la science, de la technologie et de l'innovation. Il devrait également mettre en place une structure d'intermédiation entre la recherche et le monde des entreprises, qui pourra faire le lien entre l'offre des résultats scientifiques et technologiques et leur valorisation industrielle et commerciale. L'accroissement du nombre d'infrastructures de formation au sein des universités constitue également une solution. Ce qui pourrait contribuer à accroitre la taille du secteur formel et améliorer les recettes fiscales pour stimuler l'entrepreneuriat scientifique.

Le présent travail ne se situe pas dans la lignée de ceux qui tablent sur l'impact de la recherche, dans le cadre de l'économie du savoir. Il insiste sur les préalables structurels, organisationnels pour mettre en place un système national de recherche susceptible de favoriser l'éclosion de l'entrepreneuriat scientifique au Cameroun. De ce fait, il ouvre d'autres possibilités de réflexion. On peut ainsi s'interroger sur l'impact réel de la recherche dans un tel environnement. On peut aussi s'interroger sur les stratégies des différents acteurs, notamment les chercheurs, pour pallier les insuffisances structurelles et organisationnelles du système. En effet, le fait d'avoir un système inefficace ou embryonnaire ne signifie pas nécessairement qu'il n'y a pas de recherche. Encore moins que la valorisation de la recherche est totalement absente.

\section{Bibliographie}

[AUE 03] AUERSWALD, P., BRANSCOMB, L. M., «Valleys of Death and Darwinian Seas: Financing the Invention to Innovation Transition in the United States », Journal of Technology Transfer, $n^{\circ} 28(3-4)$, p. 227-239, 2003.

[BEG 19] BEGHAIN, V., «Être étudiant entrepreneur : un levier vers l'entrepreneuriat ? Une étude de cas auprès d'étudiants entrepreneurs au sein d'écoles entrepreneuriales en Wallonie », Dynamiques régionales, $\mathrm{n}^{\circ} 7(1)$, p. 57$76,2019$.

[BEL 18] BELLACHE, K., Le rôle de l'université dans le développement de l'entrepreneuriat au Maroc, Mémoire de Master 2 Economie, conseil et gestion publique, Université de Rennes 1, 2018.

[BOU 19] BOUTILLIER S. ET ALEXANDRE L., «Editorial, l'Entrepreneuriat scientifique : institutions et innovation », Marchés et Organisations, n 34 (1), p. 11-14, 2019.

[BOU 10] BOUQUILLON, P., « Industries, économie créatives et technologies d'information et de communication », tic\&société, $\mathrm{n}^{\circ} 4$ (2), 2010.

[CIR 14] CIRAD, Etude sur l'économie de la recherche au Cameroun, MINRESI/Rapport financé par le Contrat de Désendettement et de Développement-Programme d'Appui à la Recherche.

[CHO 18] CHONWANG, J., La recherche, otage d'un manque de stratégie de vulgarisation, SciDev.Net, 2018.

[COM 03] COMMISSION DE DEONTOLOGIE DE LA FONCTION PUBLIQUE DE L'ÉTAT, $8^{\mathrm{e}}$ rapport d'activité 2002: accès des agents publics au secteur privé: rapport au Premier ministre, Paris, La Documentation française, 2003.

[COU 15] COULIBALY, B., HERMANN, H., «L'appropriation d'une innovation par ses usagers : autour du futur Learning centre de l'Université de Haute Alsace », Revue internationale de pédagogie de l'enseignement supérieur, $\mathrm{n}^{\circ} 31$ (2), [En ligne], 2015.

[DAM 2019] DAMA, D. M. « Les traits caractéristiques de la relation université-entreprise au Cameroun », Marché et Organisations, $\mathrm{n}^{\circ}$ 34(1), p. 133-157, 2019.

[DEL 10] DELPOUVE, B. ET BREILLAT, D., Country report : Cameroon », Projet Etude de Faisabilité Tuning Afrique, 2010.

[DRU 85] DRUCKER, P. F., Innovation and entrepreneurship: Practices and principles, New York: Harper \&Row, 1985.

[ETE 18] ETEKI ELOUNDOU L. S., Les enjeux de l'innovation au Cameroun, UCLGA-AFRICA, Rabat-Maroc, 2018 . 
[ETZ 08] ETZKowitz H., The Triple Helix: University-Industry-Government Innovation in Action, New York, Routledge, 2008.

[FLI 16] FLIPO, F., DELTOUR, F., DOBRE, M., « Les technologies de l'information à l'épreuve du développement durable », Natures Sciences Sociétés, n²4, p. 36-47, 2016.

[FLO 99] FLORIDA, R., « The Role of the University: Leveraging Talent, Not Technology », Issues in Science and Technology 15, $\mathrm{n}^{\circ} 4,1999$.

[FOR 18] FORTIN-LEFEBVRE, E., L'entrepreneuriat en contexte enclavé : le cas des premières Nations du Québec, Thèse de Doctorat, Université de Québec à Mont Réal, 2018.

[FOU 12] FOUDA, N. M. ET AWONO, O. C., «Les reformes de gouvernance dans l'enseignement supérieur Camerounais », Conférence de l'IIPE/UNESCO : "Réformes de gouvernance dans l'enseignement supérieur : Quelles politiques avec quels effets », Dakar, 2012.

[GAI 03] GAILLARD, J. ET ZINK, E., Les capacités de recherche scientifique au Cameroun. Une évaluation de l'impact des activités de l'IFS, Rapport de recherche Nº. 5, M E S I A Etudes d'impact, 2003.

[GAS 03] GASSE, Y., «L’influence du milieu dans la création d'entreprises », Revue Organisations \& territoires, $\mathrm{n}^{\circ} 12(2)$, p. 49-56, 2003.

[GUE 1994] GUELLEC, D., KABLA, I., «Le brevet : un instrument d'appropriation des innovations technologiques », Economie et statistique, n 275(1), 83-94, 1994.

[GJE 06] GJERDING, A.N, WILDEROM P.M.C., CAMERON, S., TAYLOR, A. ET SCHEUNERT, K.-J., «L'université entrepreneuriale : vingt pratiques distinctives », Politiques et gestion de l'enseignement supérieur, $\mathrm{n}^{\circ} 18(3)$, p. $95-124,2006$.

[IND 19] INDICE MONDIAL DE L'INNOVATION, La création d'un mode de vie sain, avenir de l'innovation médicale : Principales conclusions, rapport, INSEAD/OMPI, 2019.

[INS 2011] INS, Enquête sur 1'Emploi et le Secteur Informel au Cameroun en 2011, rapport principal, phase 1, Yaoundé, 2011.

[INS 16] INS, Cameroun : le premier obstacle à l'entrepreneuriat cité par les opérateurs économiques est la fiscalité, Enquête menée auprès des entreprises camerounaises déposant une déclaration statistique et fiscale, 2016.

[KEM 12] KEMAYOU, L. R., « L’Université de Douala, entre ancrage et Désenchantement », JHEA/RESA, n 10(2), 95-117, 2012.

[KOL 2006] KOLEVA, P., NATHALIE RODET-KROICHVILI, N. ET JULIEN VERCUEIL., J., Le cahier des charges théoriques : une analyse économique évolutionnaire des institutions, Presses de l'Université de Technologie de Belfort Montbéliard, 2006.

[KIT 05] KITGAWA, F., «Universités entrepreneuriales et développement régional, Une conception territoriale de l'Europe du savoir », Politiques et gestion de l'enseignement supérieur, n 17 (3), p. 69-97, 2005.

[KRI 19] KRICHEM, K., CHABAUD, D., « une analyse comparée et critique des modèles des spin-offs univresitaires », Marchés et Organisations, n 34 (1), p. 61-86, 2019.

[LAM 06] LAMY E. ET SHINN T., «L'autonomie scientifique face à la mercantilisation », Actes de la recherche en sciences sociales, $\mathrm{n}^{\mathrm{o}} 164$, p. 23-50, 2006.

[LOC 05] LOCKETT, A., WRIGHT, M., Resources, Capabilities, Risk Capital and the Creation of University SpinOut Companies, Research Policy, n 34, p. 1043-1057, 2005.

[MAI 97] MAINGARI, D., « La professionnalisation de l'enseignement au Cameroun : des sources aux fins », dans A. GONNIN-BOLO et G. BAILLAT (dir), L'identité enseignante : entre formation et activité professionnelle, Recherche \& Formation, n²5. p. 97-112, 1997.

[MAR 05] MARKMAN, G. D., GIANIODIS, P. T., PHAN, P. H., BALKIN, D. B., « Innovation Speed: Transferring University Technology to Market », Research Policy, n 34, p. 1058-1075, 2005.

[MAT 15] MATT, M. ET SCHAEFFER, V., « Le soutien à l'entrepreneuriat académique dans le modèle d'université hub », Innovations, n48(3), p. 13-39, 2015.

[MBA 12] MBANG, C.E., Appropriation d'outils technologiques par les acteurs : le cas des entreprises du secteur financier au Cameroun, Gestion et management, Université Paris-Est, Fr., 2012. 
[MBO 04] MBOCK, C. G., NGO-MPECK, M-L, KOM, D. ET ZAMBO BELINGA, J.M., « Utilisation des résultats de la recherche dans l'action publique au Cameroun », Revue internationale des sciences sociales, $\mathrm{n}^{\circ} 179$ (1), p. 43 $51,2004$.

[MES 19] MESRI, « Dépenses de recherche et développement en France. Résultats détaillés pour 2016 et premières estimations pour $2017 »$, note d'information $n^{\circ} 19(01), 2019$.

[MIN 14] MINISTERE DE L'ECONOMIE, Suivi et évaluation de l'état de mise en œuvre du document de stratégie pour la croissance et l'emploi-volet emploi, Rapport sur le dispositif de suivi de l'emploi, 2014.

[MOT 98] MOTHE, C., L'appropriation de ressources technologiques et scientifiques », VIIe Conférence AIMS, Louvain,1998.

[NGW 16] NGWE, L., «D'un système voué à la construction de l'Etat au projet d'un modèle entrepreneurial de développement : les métaphores de l'enseignement supérieur au Cameroun », dans NGWE, L. et H. de P. POKAM (dir), Enseignement supérieur au Cameroun depuis la réforme de 1993. Dynamiques et perspectives, CODESRIA, DAKAR, 2016.

[NSA 13] NSAN A NSAN, D., Etude diagnostique du secteur jeunesse/emploi : Communes de Bangangté, Mbouda et Nkongsamba $1^{\text {er }}$, Rapport d'étude sectorielle du projet de promotion de la gouvernance locale (PGL/OL), 2013.

[O'SH 05] O'SHEA, R. P., ALLEN, T. J., CHEVALIER, A., ROCHE, F. (2005), « Entrepreneurial Orientation, Technology Transfer and Spinoff Performance of US Universities », Research Policy, n 34, p. 994-1009.

[OCD 12] OCDE, Promouvoir l'entrepreneuriat dans les universités tunisiennes, Documents de travail, $n^{\circ} 18$, OCDE, 2012.

[RAJ 11] RAJHI, N., Conceptualisation de l'esprit entrepreneurial et identification des facteurs de son développement à l'université, Thèse de doctorat, Université de Grenoble, 2011.

[SHI 05] SHINN, T., RAGOUET, P. R. «Controverses sur la science: pour une sociologie transversalité de l'activité scientifique », Raisons d'agir, Paris, 2005.

[TCH 11] TCHOUATA, F.C., TCHOKOTE, E.C., LAMAGO, F.M., SINGO N.C., Environnement psychosocial et fraude aux examens universitaires, Rapport financé par le Réseau Ouest et Centre Africain de Recherche en Education (ROCARE), 2011.

[UZU 18] UZUNIDIS, D., "Recherche académique et innovation. La force productive de la science », dans D. UZUNIDIS (dir), Collections. Business and innovation, Peter Lang, Suisse, 2018.

[WRI 12] WRIGHT, M., CLARYSSE, B., MOSEY, S., Strategic Entrepreneurship, Resource Orchestration and Growing Spin-Offs From Universities, Technology Analysis \& Strategic Management, n 24(9), p. 911-927, 2012. 